\title{
Nuclear uncertainties in the spin-dependent structure functions for direct dark matter detection
}

\author{
D. G. Cerdeño, ${ }^{1,2}$ M. Fornasa, ${ }^{3}$ J.-H. Huh, ${ }^{1,2}$ and M. Peiró ${ }^{1,2}$ \\ ${ }^{1}$ Instituto de Física Teórica, UAM/CSIC, Universidad Autónoma de Madrid, Cantoblanco, E-28049 Madrid, Spain \\ ${ }^{2}$ Departamento de Física Teórica, Universidad Autónoma de Madrid, Cantoblanco, E-28049 Madrid, Spain \\ ${ }^{3}$ School of Physics and Astronomy, University of Nottingham, University Park NG7 2RD, United Kingdom
}

(Received 11 September 2012; published 18 January 2013)

\begin{abstract}
We study the effect that uncertainties in the nuclear spin-dependent structure functions have in the determination of the dark matter (DM) parameters in a direct detection experiment. We show that different nuclear models that describe the spin-dependent structure function of specific target nuclei can lead to variations in the reconstructed values of the DM mass and scattering cross section. We propose a parametrization of the spin structure functions that allows us to treat these uncertainties as variations of three parameters, with a central value and deviation that depend on the specific nucleus. The method is illustrated for germanium and xenon detectors with an exposure of $300 \mathrm{~kg} \mathrm{yr}$, assuming a hypothetical detection of DM and studying a series of benchmark points for the DM properties. We find that the effect of these uncertainties can be similar in amplitude to that of astrophysical uncertainties, especially in those cases where the spin-dependent contribution to the elastic scattering cross section is sizable.
\end{abstract}

DOI: 10.1103/PhysRevD.87.023512

PACS numbers: $95.35 .+\mathrm{d}$

\section{INTRODUCTION}

Direct searches of dark matter (DM) aim to observe this abundant but elusive component of the Universe by detecting its recoils off target nuclei of a detector (for a recent review, see, e.g., Ref. [1]). A large number of experiments have been taking data in the last decades or are currently under construction with this objective, leading to a very exciting present situation.

In fact, some experiments have claimed potential signals that could be compatible with the detection of a weakly interacting massive particle (WIMP). This is the case of the DAMA Collaboration [2], which observed an annual modulation in the recoil rate on a NaI target that was later confirmed by the upgraded DAMA/LIBRA detector [3]. Similarly, the CoGeNT Collaboration, with a germanium target, reported an irreducible excess in their data that could point towards very light WIMPs [4], and also observed an annual modulation effect [5], although the latter is not easy to reconcile with the DAMA/LIBRA result. Finally, the CRESST-II experiment, which uses $\mathrm{CaWO}_{4}$ as a target, also reported an excess [6] over the expected background. However, these observations are in conflict with the negative results obtained in searches by other experimental collaborations. Experiments such as CDMS-II [7,8], XENON10 [9], XENON100 [10,11], EDELWEISS [12], SIMPLE [13], KIMS [14], and a combination of CDMS and EDELWEISS data [15] are in strong tension with the regions of the parameter space compatible with WIMP signals in DAMA/LIBRA or CoGeNT. Moreover, a reanalysis of CDMS data has been performed in order to look for annual modulation with negative results [16].

The elastic scattering cross section of WIMPs off nuclei can be separated into two components, spin-independent
(SI) $\sigma_{0}^{\mathrm{SI}, N}$ and spin-dependent (SD) $\sigma_{0}^{\mathrm{SD}, N}$, which originate from different terms in the Lagrangian describing the interaction of a DM particle with quarks. The SI term stems from scalar or vector couplings, and its contribution to the total WIMP-nucleus cross section scales as the nucleon number squared, $A^{2}$; the $\mathrm{SD}$ term originates from axial-vector couplings, and its total contribution to the cross section off nuclei is only a function of the total nuclear angular momentum and the DM spin. Thus, the SI term typically dominates for heavy nuclei.

Constraints are normally expressed in terms of the SI and SD components of the WIMP-nucleon elastic cross section, $\sigma^{\mathrm{SI}}$ and $\sigma^{\mathrm{SD}}$, respectively. To date, the most stringent constraints on $\sigma^{\text {SI }}$ are those obtained from the XENON100 data [11] that exclude SI cross sections above $\sigma^{\mathrm{SI}} \approx 2 \times 10^{-8} \mathrm{pb}$ for a mass around $50 \mathrm{GeV}$, as well as XENON10 [9] and the low-energy reanalysis of CDMS-II [8], which dominate for light WIMPs. Regarding the SD contribution, the leading bounds from direct detection experiments have been provided by XENON [17] (SD cross section with neutrons, $\left.\sigma^{\mathrm{SD}, n}\right)$ and COUPP [18] and PICASSO [19] (SD cross section with protons, $\sigma^{\mathrm{SD}, p}$ ), but indirect detection experiments such as SuperKamiokande [20] and IceCube [21], as well as searches for mono-jet $[22,23]$ and mono-photon plus missing energy at the Tevatron [24] and LHC [25-27], lead to even more compelling constraints on $\sigma^{\mathrm{SD}, p}$. Larger and more sophisticated direct detection experiments are currently under development that will be able to explore the DM parameter space with unprecedented sensitivity. This is the case, for example, of the SuperCDMS and XENON1T collaborations, which aim at the construction of 1 ton scale detectors based on germanium and xenon, respectively.

In the light of this promising experimental situation, it seems plausible that the DM can be discovered in the near 
future in direct detection experiments. In such an event, the study of the signal rate and spectrum (differential rate) can be used to determine some of the DM properties, namely, its mass $m_{\chi}$ and elastic scattering cross section [28-30]. The precision of this reconstruction is very sensitive to the characteristics of the detector and is affected by uncertainties in the parameters describing the DM halo, as well as in the nuclear form factors. Astrophysical uncertainties have been widely discussed in the literature [31-34], and they are known to introduce significant errors in the determination of the mass and scattering cross section of DM. Regarding nuclear uncertainties, those in the SI form factor have been argued to be relatively small [35]. The effect of variations in the SD form factors has not been previously addressed and constitutes the objective of this work.

We consider the hypothetical future observation of a DM candidate in a direct detection experiment and, sampling over the three-dimensional space of $\left(m_{\chi}, \sigma^{\mathrm{SI}}, \sigma^{\mathrm{SD}}\right)$, we investigate how the reconstruction of these quantities is affected by nuclear uncertainties in the spin-dependent structure function of the target nucleus. In order to do so, we propose a description of structure functions based on three parameters, which enlarge the parameter space sampled, and allow us to incorporate uncertainties in a consistent and systematic way. This provides a general method, applicable to any detector target. We particularize our analysis for the case of a germanium detector (such as, e.g., SuperCDMS), for which we consider the spindependent structure functions provided by the analysis of various groups [36-38], and for xenon detectors (such as, e.g., the future XENON1T), for which we use the structure functions derived in Refs. [39,40].

We observe that the effect of nuclear uncertainties in SD structure functions can lead to variations in the reconstructed DM mass and SD elastic cross section, the effect being more important in those scenarios in which the SD term in the WIMP-nucleus cross section is the main contribution to the total detection rate. In such cases uncertainties in the spin-dependent structure functions are similar in amplitude to those induced by astrophysical uncertainties in the DM halo parameters, although the latter also affect the SI component.

The paper is organized as follows. In Sec. II we introduce the formalism used to compute the recoil event rate, emphasizing the role of SD interactions. We concentrate on the case of a germanium detector, introduce the models available in the literature that describe the spin-dependent structure function, and comment on their differences. Section III describes the generation of the simulated data for a set of benchmark models, and the implementation of the scanning algorithm to probe the phenomenological parameter space. In Sec. IV we show the reconstruction of DM parameters for each benchmark scenario, using different nuclear models for the SD structure function and investigating how this alters the predictions for the
DM properties. In Sec. V we present a parametrization of the SD structure function that allows us to systematically account for uncertainties when scanning over our parameter space, and we apply the method to the cases of germanium and xenon detectors. Our conclusions are summarized in Sec. VI.

\section{NUCLEAR UNCERTAINTIES IN DIRECT DARK MATTER DETECTION}

The differential event rate for the elastic scattering of a WIMP with mass $m_{\chi}$ off a nucleus with mass $m_{N}$ is given by [41]

$$
\frac{d R}{d E_{R}}=\frac{\rho_{0}}{m_{N} m_{\chi}} \int_{v_{\min }}^{v_{\mathrm{esc}}} v f(v) \frac{d \sigma}{d E_{R}}\left(v, E_{R}\right) d v
$$

where $\rho_{0}$ is the local WIMP density and $f(v)$ is the WIMP velocity distribution in the detector frame normalized to unity. The integration over the WIMP velocity $v$ is performed from the minimum needed to induce a recoil of energy $E_{R}, v_{\min }=\sqrt{m_{N} E_{R} / 2 \mu_{N}^{2}}$, to the escape velocity $v_{\text {esc }}$ above which WIMPs are not bound to the Milky Way. The WIMP-nucleus elastic scattering cross section $d \sigma / d E_{R}$ is expressed as a function of the recoil energy, and $\mu_{N}=m_{N} m_{\chi} /\left(m_{N}+m_{\chi}\right)$ is the reduced mass. The total event rate is calculated by integrating Eq. (1) over all the possible recoil energies in a window defined by a threshold energy $E_{T}$ and a maximal energy $E_{\max }$, both depending on the experiment. ${ }^{1}$

In general, the WIMP-nucleus cross section is separated into a SI and a SD contribution, as follows:

$$
\frac{d \sigma}{d E_{R}}=\frac{m_{N}}{2 \mu_{N}^{2} v^{2}}\left(\sigma_{0}^{\mathrm{SI}, N} F_{\mathrm{SI}}^{2}\left(E_{R}\right)+\sigma_{0}^{\mathrm{SD}, N} F_{\mathrm{SD}}^{2}\left(E_{R}\right)\right),
$$

where $\sigma_{0}^{\mathrm{SI}, N}$ and $\sigma_{0}^{\mathrm{SD}, N}$ are the SI and SD WIMP-nucleus cross sections at zero momentum transfer. $F_{\mathrm{SI}}\left(E_{R}\right)$ and $F_{\mathrm{SD}}\left(E_{R}\right)$ are the SI and SD form factors that account for the coherence loss which leads to a suppression of the event rate for heavy WIMPs or heavy nuclei. See Ref. [41] for a complete description of these prescriptions. The differential rate $d R / d E_{R}$ depends on the recoil energy $E_{R}$ through the form factors and the minimal velocity $v_{\text {min }}\left(E_{R}\right)$.

The total number of recoils, as well as their distribution in energy, are affected by uncertainties in the nuclear form factors (both SI and SD) and in the parameters describing the DM halo (usually referred to as astrophysical uncertainties). Determining the impact of these is crucial to understand the capability of a DM experiment to reconstruct the properties of the WIMP.

\footnotetext{
${ }^{1}$ In order to take into account the energy resolution of the detector, the differential rate is convoluted with a Gaussian, whose standard deviation is a function of the recoil energy, as done in Ref. [42].
} 
The role of astrophysical uncertainties has been widely addressed in the literature. They are known to significantly affect the reconstruction of both the mass and scattering cross section of the DM [31,33,43-46]. Since the subject of our work is to study the effect of nuclear uncertainties from the form factors, we do not include astrophysical ones. We therefore consider a fixed model for the DM halo, namely, the standard halo model with an escape velocity of $v_{\text {esc }}=$ $544 \mathrm{~km} \mathrm{~s}^{-1}$, a central velocity $v_{0}=230 \mathrm{~km} \mathrm{~s}^{-1}$ [47-51], and a local dark matter density $\rho_{0}=0.4 \mathrm{GeV} \mathrm{cm}^{-3}$ [52-55].

\section{A. Uncertainties in the SI form factors}

Regarding SI interactions, the so-called Woods-Saxon form factor is the Fourier transform of the nucleon distribution function $\rho_{A}(x)$,

$$
F_{\mathrm{SI}}(q)=\int e^{-i q x} \rho_{A}(x) d^{3} x,
$$

where $q=\sqrt{2 m_{N} E_{R}}$ is the momentum transfer. The Fermi distribution is assumed for the nucleon distribution,

$$
\rho_{A}(x) \propto \frac{1}{1+\exp \left[\left(r-R_{A}\right) / a\right]},
$$

where $R_{A}=\left(1.23 A^{1 / 3}-0.6\right) \mathrm{fm}, A$ is the nucleon number, and $a=0.5 \mathrm{fm}$ the surface thickness of the nucleus. Other parametrizations can be found in the literature. In Ref. [56] it was shown that differences may exist, even at relatively low momentum transfers, between generic form factors such as the Helm model and more realistic modelindependent calculations which use charge densities derived from elastic electron scattering. However, the Wood-Saxon form factor provides a good description of the nuclear structure for energies in the range between 1 and $100 \mathrm{keV}$, typical of WIMP scatterings. Similarly, it has been shown in Ref. [35] that the differences in the SI form factors due to small deformations of the nuclei can be safely neglected. In fact, we have explicitly checked that this is indeed the case when using realistic nuclear density profiles obtained from a state-of-the-art mean field calculation. Thus, throughout this paper we consider the form factor in Eq. (3) with no associated uncertainty.

\section{B. Uncertainties in the SD form factors}

On the other hand, the effect of uncertainties in the SD form factors has not been addressed in the literature. The SD contribution to the WIMP-nucleus differential cross section in Eq. (2) can be expanded as a function of the WIMP couplings to the matrix elements of the axial-vector currents in protons $\left(a_{p}\right)$ and neutrons $\left(a_{n}\right)$,

$$
\begin{aligned}
\left(\frac{d \sigma}{d E_{R}}\right)_{\mathrm{SD}}= & \frac{16 G_{F}^{2} m_{N}}{\pi v^{2}} \frac{(J+1)}{J}\left(a_{p}\left\langle S_{p}\right\rangle\right. \\
& \left.+a_{n}\left\langle S_{n}\right\rangle\right)^{2} F_{\mathrm{SD}}^{2}\left(E_{R}\right),
\end{aligned}
$$

where $J$ is the total spin of the nucleus and $\left\langle S_{p}\right\rangle\left(\left\langle S_{n}\right\rangle\right)$ is the proton (neutron) spin averaged over the nucleus. The SD form factor $F_{\mathrm{SD}}^{2}\left(E_{R}\right)=S\left(E_{R}\right) / S(0)$ is commonly expressed as a decomposition into isoscalar $\left(a_{0}=a_{p}+a_{n}\right)$ and isovector $\left(a_{1}=a_{p}-a_{n}\right)$ couplings,

$$
S(q)=a_{0}^{2} S_{00}(q)+a_{0} a_{1} S_{01}(q)+a_{1}^{2} S_{11}(q),
$$

where $q$ is the momentum transfer. The quantities $S_{00}(q)$, $S_{11}(q)$, and $S_{01}(q)$ are the spin-dependent structure functions (SDSFs) and are computed using nuclear physics models, whereas the couplings $a_{p}$ and $a_{n}$ (and consequently $a_{0}$ and $a_{1}$ ) are specific to the particle physics model for DM and are computed from the diagrams describing the WIMP-nucleon interaction. In order to continue with a model-independent approach $^{2}$ we assume a specific relation between $a_{p}$ and $a_{n}$, and consider the cases $a_{p} / a_{n}= \pm 1$. Under this assumption, ${ }^{3}$ Eq. (5) reduces to

$$
\left(\frac{d \sigma}{d E_{R}}\right)_{\mathrm{SD}}= \begin{cases}\frac{64 G_{F}^{2} m_{N}}{v^{2}(2 J+1)} a_{p}^{2} S_{00}(q) & \frac{a_{p}}{a_{n}}=1, \\ \frac{64 G_{F}^{2} m_{N}}{v^{2}(2 J+1)} a_{p}^{2} S_{11}(q) & \frac{a_{p}}{a_{n}}=-1 .\end{cases}
$$

The SDSFs $S_{00}(q)$ and $S_{11}(q)$ can be calculated using a shell-model (ShM) description of the atomic nucleus, where the nuclear spin properties are obtained by the wave functions of a few valence nucleons, those which do not cancel out the spin of the nucleus in pairs. In particular, $S_{00}(q)$ and $S_{11}(q)$ are related to the transverse electric and longitudinal projections of the axial current. To calculate these quantities in the ShM, the nucleons are placed in energy levels according to the exclusion principle, assuming a particular interaction between nucleons (typically a harmonic oscillator potential) and including as many excited states as possible, making this kind of calculation very difficult.

ShM calculations are generally more reliable for heavy nuclei than for light ones. The same holds for nuclei close to magic numbers, elements featuring closed shells being more easily modeled. An example is ${ }^{19} \mathrm{~F}$, which has 9 protons and 10 neutrons, thus only one proton above a magic number. On the other hand, the nucleus of ${ }^{73} \mathrm{Ge}$ is much more difficult to model since it has 32 protons and 41 neutrons, the nearest closed shell being the one with 28 nucleons. In this case, deviations of the real nucleus from the ShM should be expected, as well as differences in the

\footnotetext{
${ }^{2} \mathrm{~A}$ prescription independent of the DM model is possible at zero momentum transfer [57]. Also, it has been argued that the similar momentum dependence of $S_{i j}(q)$ can be used [58] to extract a common form factor.

${ }^{3}$ This is equivalent to reducing by 1 the dimensionality of our parameter space, assuming a relation between $\sigma^{\mathrm{SD}, p}$ and $\sigma^{\mathrm{SD}, n}$. Our analysis can easily be extended to consider the full fourdimensional parameter space $\left(m_{\chi}, \sigma^{\mathrm{SI}}, \sigma^{\mathrm{SD}, p}, \sigma^{\mathrm{SD}, n}\right)$, but this renders the discussion more cumbersome. Furthermore, particle models for DM generally predict $\left|\sigma^{\mathrm{SD}, n}\right| \approx\left|\sigma^{\mathrm{SD}, p}\right|$.
} 

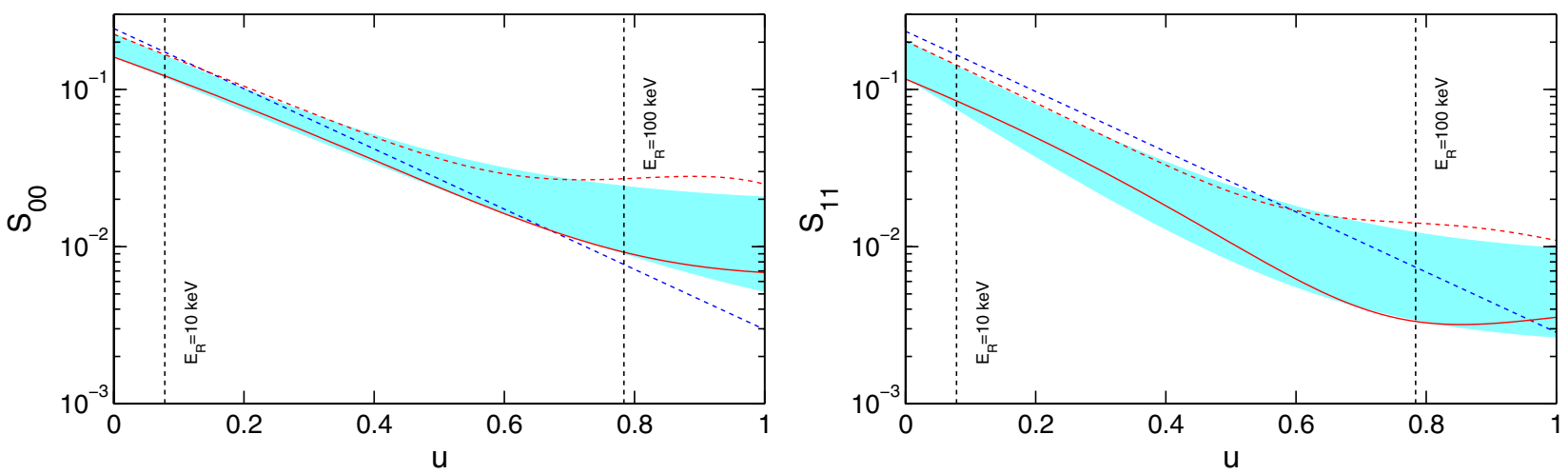

FIG. 1 (color online). Spin-dependent structure functions as a function of $u$, in the case of $a_{p} / a_{n}=1$ (left panel) or $a_{p} / a_{n}=-1$ (right panel). The solid (dashed) red lines correspond to the D model [37] (R model [38]), and the dotted blue line indicates the Gaussian approximation of Eq. (8). The blue region covers the area spanned by the family of curves in Eq. (14). The vertical black dashed lines indicate the WIMP search window used in the analysis.

results when different ShMs are used. In the first part of the paper we consider the case of germanium, for which the only natural isotope that contributes to the SD cross section is ${ }^{73} \mathrm{Ge}$.

In the case of ${ }^{73} \mathrm{Ge}$, various ShM calculations are available in the literature. We consider two different, commonly used parametrizations, from Ressel et al. [37] and Dimitrov et al. [38], which we refer to as R and D models, respectively. They differ in the methodology and in the choice of the nuclear interaction potential, but both reproduce the value of the magnetic momentum of ${ }^{73} \mathrm{Ge}$. The SDSFs in both cases can be expressed as a function of the adimensional quantity $u$, related to the momentum transfer as $u=$ $(q b)^{2} / 2$, where $b$ is the oscillator size parameter, $b=A^{1 / 6}$.

The SDSFs for the R and D models are plotted as a function of $u$ in Fig. 1 by means of red dashed and solid red lines, respectively. The left (right) panel refers to the case $a_{p} / a_{n}=1\left(a_{p} / a_{n}=-1\right)$. The vertical, black dashed lines indicate the values of $u$ that correspond to the WIMP search window that we use in our analysis, from a threshold energy of $10 \mathrm{keV}$, to an energy of $100 \mathrm{keV}$ (as currently done in CDMS-II). The dotted blue lines indicate a Gaussian approximation [see Eq. (8) below]. Finally, the blue areas represent the regions spanned by a family of curves, obtained by a parametrization which interpolates between the $\mathrm{R}$ and $\mathrm{D}$ models that will be introduced in Sec. V.

The two SDSFs differ in the zero momentum value (the $\mathrm{R}$ model being larger for the whole energy range of interest for direct detection), and also in the shape at large energies. They both start as decreasing power laws at low-energy flattening out as $u$ increases. However, the transition happens sooner for the $\mathrm{R}$ model (around $u=0.5$ ) than for the $\mathrm{D}$ model. The slope for the $\mathrm{D}$ model is also slightly steeper than for the R model, especially in $S_{11}(q)$. As we will see in Sec. IV these differences play an important role when determining the DM parameters.
There are finally some nuclei for which ShM computations of their form factors are not available. In these cases an approximation was introduced in Ref. [59] that works well in the low momentum transfer regime, but fails towards larger values of $q$,

$$
S_{i j}(q)=S(0) e^{-\frac{q^{2} R^{2}}{4}},
$$

where $R$ is an effective radius, measured in $\mathrm{fm}$, which can be written as

$R=0.92 A^{1 / 3}+2.68-0.78 \sqrt{\left(A^{1 / 3}-3.8\right)^{2}+0.2}$.

\section{DETERMINATION OF WIMP PROPERTIES}

We consider a set of benchmark scenarios (BM1, BM2, and BM3) listed in Table I that define the phenomenological DM parameters $\left(m_{\chi}, \sigma^{\mathrm{SI}}, \sigma^{\mathrm{SD}}\right)$. These benchmarks are consistent with possible particle physics models for DM. ${ }^{4}$ We then assume the observation of a DM signal in a given direct detection experiment. The differential rate is computed for each benchmark point following Eq. (1), and used to derive the total number of events $\lambda$.

We first particularize our analysis for the case of a germanium detector with a total exposure of $\epsilon=$ $300 \mathrm{~kg} \mathrm{yr}$. This could, e.g., correspond to the 1 ton phase of SuperCDMS, operating for a whole year with an efficiency of $30 \%$. We define the energy window for WIMP searches in the range $E_{T}=10 \mathrm{keV}$ and $E_{\max }=100 \mathrm{keV}$, and calculate the number of events $\left\{\lambda_{i}\right\}$ in a series of energy bins $\left\{E_{i}, E_{i}+\Delta E\right\}$ with $\Delta E=5 \mathrm{keV}$. We also include a background with a rate of $4 \times 10^{-8}$ days ${ }^{-1} \mathrm{~kg}^{-1} \mathrm{keV}^{-1}$, which is of the order of the background expected for the

\footnotetext{
${ }^{4}$ In particular, the three benchmarks can be obtained within the context of neutralino DM in the general Minimal Supersymmetric Standard Model.
} 
TABLE I. Phenomenological parameters defining the three benchmark models. We include the predicted total number of recoil events, $\lambda$, as well as the number of events (calculated using the R model) $\lambda^{\mathrm{SI}}\left(\lambda^{\mathrm{SD}}\right)$ due to SI (SD) interactions, for the experimental setup described in the text.

\begin{tabular}{lcccccr}
\hline \hline & $m_{\chi}[\mathrm{GeV}]$ & $\sigma^{\mathrm{SI}}[\mathrm{pb}]$ & $\sigma^{\mathrm{SD}}[\mathrm{pb}]$ & $\lambda$ & $\lambda^{\mathrm{SI}}$ & $\lambda^{\mathrm{SD}}$ \\
\hline BM1 & 100 & $10^{-9}$ & $10^{-5}$ & 37.2 & 36.4 & 0.8 \\
BM2 & 50 & $10^{-9}$ & $10^{-5}$ & 42.1 & 41.2 & 0.9 \\
BM3 & 100 & $10^{-9}$ & $10^{-3}$ & 79.6 & 36.4 & 43.2 \\
\hline \hline
\end{tabular}

SuperCDMS experiment in SNOLAB [60]. For the considered exposure this means a total of 0.02 background events in each of the energy bins considered (i.e., we are almost dealing with a background-free experiment). We assume that this background is flat (energy independent).

The simulated energy spectra for the three benchmark points can be seen in Fig. 2, where the solid red line corresponds to the results when the D model is used and the dashed red line is obtained for the R model. Practically no difference is observed for benchmarks BM1 and BM2. This happens because, for these two benchmark points, the main contribution to the total detection rate is due to SI interactions. On the contrary, in BM3 the SD contribution is important, and we observe how the predicted rate is significantly higher for the $\mathrm{R}$ model than for the $\mathrm{D}$ model. This is a consequence of the higher value of $S_{11}$ for the R model in the whole energy range (see Fig. 1).

We treat the quantities $\left\{\lambda_{i}\right\}$ as the experimental information from which DM parameters have to be reconstructed. Our analysis is based on the Bayes theorem, which determines the posterior probability distribution (pdf) $p(\boldsymbol{\Theta} \mid \mathbf{D})$ of a set of parameters $\boldsymbol{\Theta}$ [for which a prior probability is assumed $p(\boldsymbol{\Theta})]$ from a set of experimental data $\mathbf{D}$, encoded in the likelihood function $p(\mathbf{D} \mid \boldsymbol{\Theta})$ [or $\mathcal{L}(\boldsymbol{\Theta})$ ],

$$
p(\boldsymbol{\Theta} \mid \mathbf{D})=\frac{p(\mathbf{D} \mid \boldsymbol{\Theta}) p(\boldsymbol{\Theta})}{p(\mathbf{D})}
$$
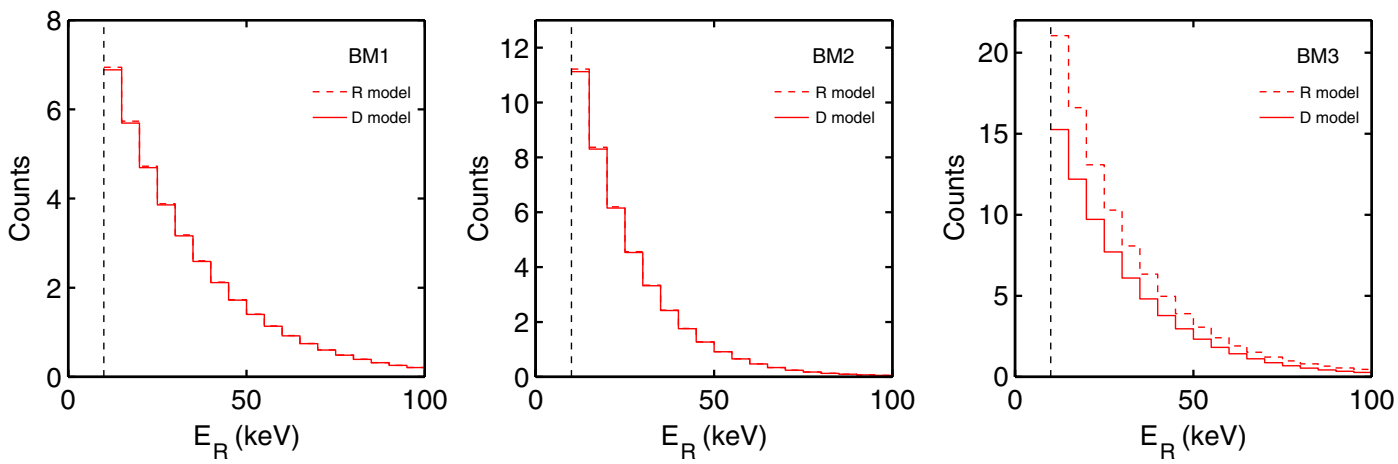

FIG. 2 (color online). Predicted DM spectra for benchmarks BM1, BM2, and BM3 (from left to right) for the experimental setup described in the text. The solid and dashed red lines correspond to the predictions using the R and D models for the SDSF, respectively. The vertical dashed line indicates the energy threshold $E_{T}$.
The evidence $p(\mathbf{D})$ in the denominator of Eq. (10) is a function of only the experimental data. For our purposes it works as a normalization factor and can therefore be ignored. The pdf in Eq. (10), in principle, depends on the priors $p(\Theta)$, and different choices of priors can affect the shape of the final pdf. However, should this happen, it would mean that the experimental data are not constraining enough and do not dominate the final probability distribution. Residual prior dependence can be seen, e.g., in Refs. [61-63]. Our scans are performed with MultiNest $2.9[64,65]$ interfaced with our own code for the computation of the number of recoil events and the likelihood. Scans are performed with 20000 live points and a tolerance of 0.0001 .

In our case, for a given benchmark point the experimental data are $\mathbf{D}=\left(\left\{\lambda_{i}\right\}\right)$, and a scan of the parameter space $\boldsymbol{\Theta}=\left(m_{\chi}, \sigma^{\mathrm{SI}}, \sigma^{\mathrm{SD}}\right)$ is performed. The ranges considered are $m_{\chi}=1-10^{5} \mathrm{GeV}, \sigma^{\mathrm{SI}}=10^{-12}-10^{-6} \mathrm{pb}$, and $\sigma^{\mathrm{SD}}=$ $10^{-8}-1 \mathrm{pb}$. Logarithmic priors are assumed for the three variables since the range scanned is quite large, spanning up to 8 orders of magnitude.

The likelihood $\mathcal{L}(\boldsymbol{\Theta})$ is calculated for each point in the scan, computing the number of recoil events $N_{i}$ in the $i$ th bin, and comparing it with the prediction of the benchmark model in the same bin, $\lambda_{i}$, assuming that all experimental data follow an independent Poissonian distribution,

$$
\mathcal{L}(\Theta)=\prod_{i} \frac{N_{i}(\boldsymbol{\Theta})^{\lambda_{i}} e^{N_{i}(\boldsymbol{\Theta})}}{\lambda_{i} !} .
$$

The number of recoil events $N_{i}$ in the $i$ th bin are obtained by integrating Eq. (1) between $E_{i}$ and $E_{i}+\Delta E$, and including a certain number of background events $b_{i}$. The latter is included as a nuisance parameter in our scans, following a Poissonian distribution function with a mean

The results of our scans are plotted in the next sections by means of one- or two-dimensional plots. When the probability for a subset of the original $\boldsymbol{\Theta}$ is considered, value of 0.02 . 
one can account for the presence of the hidden parameters in two different ways:

(i) by marginalizing over them, obtaining the pdf for the $j$ th parameter integrating over all the others,

$$
p\left(\Theta_{j} \mid \mathbf{D}\right)=\int p(\boldsymbol{\Theta} \mid \mathbf{D}) d \Theta_{1}, \ldots, d \Theta_{j-1} d \Theta_{j+1} d \Theta_{n} ;
$$

(ii) by maximizing over them, obtaining the so-called profile likelihood

$$
\mathcal{L}\left(\Theta_{j}\right)=\max _{\Theta_{1}, \ldots, \Theta_{j-1}, \Theta_{j+1}, \Theta_{n}} \mathcal{L}(\boldsymbol{\Theta}) .
$$

The profile likelihood is usually more sensitive to small fine-tuned regions with large likelihood, while the integration implemented for the pdf accounts for volume effects. Thus, a parameter space characterized by a complicated likelihood function may result in different pdf and profile likelihood distributions for the same parameter. In the following we will present plots for both the pdf and the profile likelihood since they have different statistical meanings and provide complementary information.

It was recently pointed out in Ref. [34] that the method outlined here is affected by an intrinsic statistical limitation associated with the fact that only one set of simulated data is obtained for each benchmark point. In our work we do not incorporate this effect, since we want to isolate the variations due to nuclear uncertainties. Furthermore, our benchmark points are in regions with good coverage (see Figs. 2 or 4 in Ref. [34]), and the number of events is relatively large.

\section{RESULTS}

We begin by considering the three benchmarks BM1, $\mathrm{BM} 2$, and BM3, with the total rate and energy spectrum of nuclear recoils as given in Table I. For concreteness we consider explicitly the case $a_{p} / a_{n}=-1$, which implies looking at only the $S_{11}$ component of the SDSF, according to Eq. (7). This would correspond, e.g., to a DM candidate whose SD interaction is dominated by $Z$ exchange [66]. Other choices of $a_{p} / a_{n}$ lead to qualitatively similar results (but then a linear combination with the other components, $S_{00}$ and $S_{01}$, also appears). We emphasize at this point that a full analysis can be done without fixing this ratio and including a fourth parameter in the scan; however, this makes it more difficult to isolate the effects of uncertainties in the SDSF.

As a first exercise in parameter reconstruction, we assume (as it is often done) that the SD contribution is vanishing, and we attempt to reconstruct the WIMP mass and SI cross section from the experimental data (of course, this assumption is not made when preparing the simulated data from the benchmark points).
The resulting two-dimensional profile likelihood for these quantities ${ }^{5}$ is given in Fig. 3. An obvious thing to observe is that the reconstruction of these two parameters is good for benchmarks BM1 and BM2, since in these cases the SI contribution is the dominant term in the detection rate. This is obviously not the case in BM3, where SD interactions play a more important role. For this benchmark point, ignoring the SD contribution term leads to an overestimation of the SI-independent cross section of approximately a factor 2 (in order to account for the total detected rate).

Another feature that can be observed, and is consistent with the existing literature, is that the goodness of the reconstruction is very dependent on the mass of the DM candidate $[28,29]$ (see also Refs. [34,42,67]). In particular, we can see how in benchmark BM1 the 99\% confidence level contours are open for heavy WIMPs, whereas this is not the case for BM2 and BM3. In principle, increasing the DM mass makes the recoil energy spectrum flatter, as a consequence of the dependence of $v_{\text {min }}$ which enters through the reduced mass. Thus, one expects to produce a worse fit to the recoil spectrum, as we scan more massive DM candidates. In benchmarks BM2 and BM3 this is the reason why heavy masses are disfavored; however, in BM1 the number of events in each energy bin is too low to pick up this tendency, and very massive DM candidates can still produce a good fit to the data. The presence of a flat background also enhances this effect. On the other hand, the contours do not extend to low WIMP masses because particles with masses below $m_{\chi} \sim 30-40 \mathrm{GeV}$ produce a much steeper spectrum. Notice finally that the assumption $\sigma^{\mathrm{SD}}=0$ leads to a lower limit for the SI cross section that allows us to reconstruct the value of $\sigma^{\text {SI }}$ up to approximately a factor 5 (for a fixed value of DM mass).

An unbiased reconstruction of DM parameters, however, has to include the possibility that $\sigma^{\mathrm{SD}} \neq 0$. In fact, when we allow for a non-negligible SD contribution to the WIMP scattering cross section, we find that a new degeneracy in the parameter space arises: the same detected rate can be explained by a DM with either pure SD or pure SI interactions or, in general, a given combination of both as we see from Eqs. (1) and (2). This implies that the closed contours in Fig. 3 can extend towards arbitrarily small values of $\sigma^{\mathrm{SI}}$. It is when we acknowledge this possibility that uncertainties in the SDSF play a nontrivial role, as they affect the total rate and energy spectrum of WIMP recoils.

Choosing germanium as a case study, we consider the two calculations, R and D models, for the SDSF of ${ }^{73} \mathrm{Ge}$ (the isotope that contributes to the SD cross section) that were introduced in Sec. IIB, performing the Bayesian inference for both SDSFs. Strictly speaking, we have to

\footnotetext{
${ }^{5}$ Note that, since we are scanning over only two parameters, there is no need for marginalization or maximization.
} 

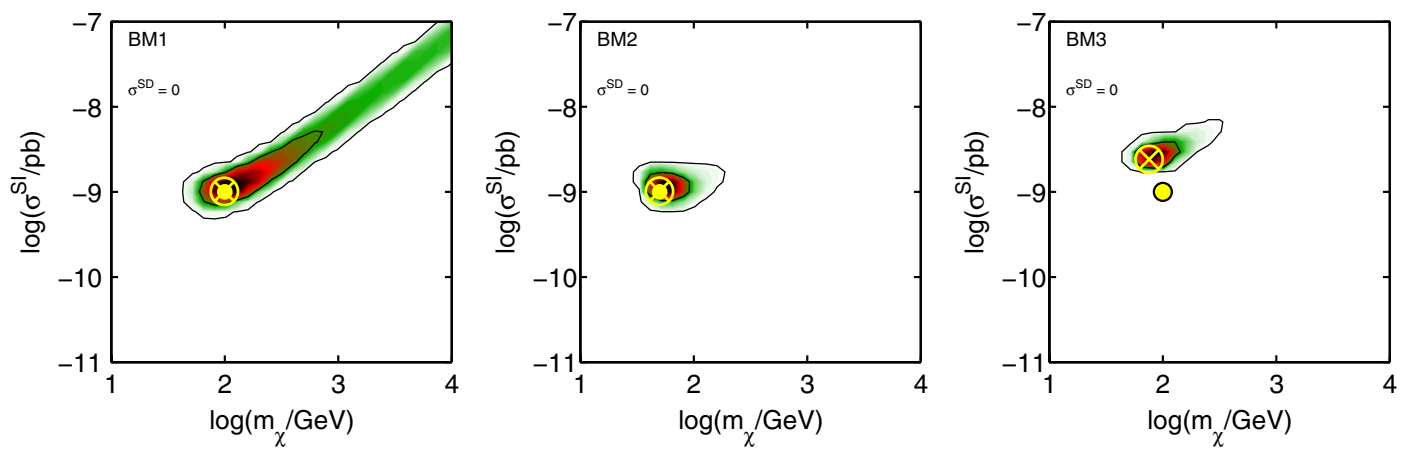

FIG. 3 (color online). Two-dimensional profile likelihood in the plane $\left(m_{\chi}, \sigma^{\mathrm{SI}}\right)$ for benchmarks BM1, BM2, and BM3, from left to right, assuming in the reconstruction that $\sigma^{\mathrm{SD}}=0$. The inner and outer contours are 68 and $99 \%$ confidence level regions, respectively. The yellow dot indicates the benchmark values in each case, and the encircled yellow cross marks the positions of the best-fit point.

select one SDSF from which the simulated data for a given benchmark are generated and, then, one SDSF for the computation of the likelihood in Eq. (11) at the moment of the scan. The two choices are independent, leaving us with four possibilities of combining the two SDSFs. In particular, we can either use the same (R or D) model for the generation of the simulated experimental data and for the parameter reconstruction, or we can generate the data with one model and perform the reconstruction with the other one. This last possibility gives an idea on how sizable the misreconstruction of DM parameters can be if we use an incorrect model for the SDSF, that is, one different from the real one. We perform this computation for the three benchmark points.

The results for the first benchmark, BM1, are displayed in Fig. 4, where we show both the profile likelihood and marginalized pdf. Comparing the distribution of the profile likelihood with that of the pdf, we can observe the effect of maximization versus marginalization. There are regions of the parameter space that are contained in the 99\% confidence level contour of the profile likelihood, which are however left out of the credible interval contours of the pdf. This happens because the good agreement with the data is produced only in a small volume of the three-dimensional parameter space, and the integration in the third dimension decreases the corresponding value for the pdf. ${ }^{6}$

The plots in the first column of Fig. 4 represent the reconstruction of the WIMP mass and SI cross section, and they can therefore be compared with the leftmost plot of Fig. 3. We observe that $\sigma^{\text {SI }}$ can now be arbitrarily small as long as the $\sigma^{\mathrm{SD}}$ is large enough to reproduce the observed DM rate and that the assumption $\sigma^{\mathrm{SD}}=0$ leads to over-constrained contours. The SD form factor (that

\footnotetext{
${ }^{6}$ The contours in the two-dimensional plots for the profile likelihood look smoother than for the pdf. This occurs, in particular, when integrating over regions with an almost flat likelihood, where it is difficult to obtain uniform sampling. The resulting pdf can present unphysical structures. The fact that the background is included as a free parameter in the scan introduces additional fluctuations.
}

results for both R and D models of the SDSF) is steeper than the SI form factor. Thus, in principle, using the information from the energy spectrum, it would not be possible to properly fit BM1 data with only SD interactions (large values of the WIMP mass provide, in this case, a better fit, since they would correspond to a flatter energy spectrum). However, the number of recoil events in the high energy bins is too small to be sensitive to those differences.

Similarly, there is no lower bound for the SD cross section. This is evidenced in the second column of Fig. 4 , where $\sigma^{\mathrm{SD}}$ is plotted as a function of the WIMP mass. As commented above, when trying to fit the energy spectrum of a point dominated by SI interactions (such as BM1) in terms of axial interactions, we get better results for large WIMP masses, due to the SDSF being steeper. The trend is evident in the pdf plots where larger values of masses are associated with a brighter pdf than lower masses, but the small number of events prevents this tendency from having any significant effect on the shape of the contours.

Finally, the degeneracy in the reconstruction of the SI and SD contributions for a given set of experimental data is clearly evidenced in the third column of Fig. 4, where $\sigma^{\mathrm{SD}}$ is plotted versus $\sigma^{\mathrm{SI}}$ and the resulting compatible regions show an "inverse L" pattern. The lower left corner of the plot is empty since both the SD and SI cross sections are too small to produce the simulated number of nuclear recoils; however, as stressed in the previous paragraphs, both $\sigma^{\mathrm{SI}}$ and $\sigma^{\mathrm{SD}}$ can be sizable if the WIMP mass is also large. These plots also show that for this particular benchmark point, SD interactions provide the dominant contribution to the WIMP rate for $\sigma^{\mathrm{SD}} \geq 10^{-3} \mathrm{pb}$.

The interpretation of the results for the different rows allows us to determine to what extent the uncertainties in the SDSF affect the reconstruction of DM parameters. We stress again that for BM1 the differential event rate is dominated by SI interactions; thus, we do not observe significant differences when changing the SDSF in the computation of the simulated recoils (see the left panel in 

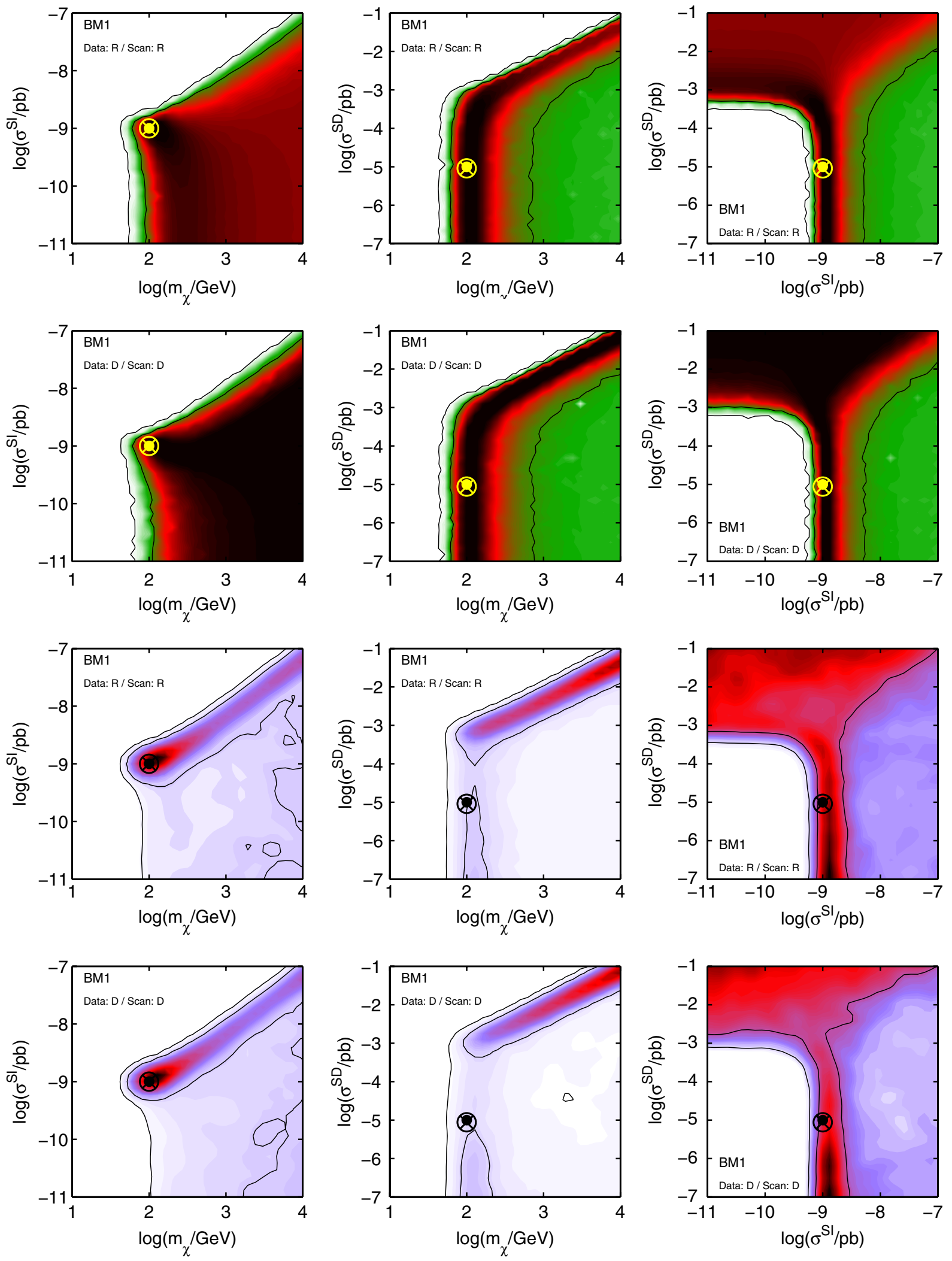

FIG. 4 (color online). Two-dimensional profile likelihood for the reconstructed parameter space $\left(m_{\chi}, \sigma^{\mathrm{SI}}, \sigma^{\mathrm{SD}}\right)$ in benchmark model BM1. The model used for the SDSF in the simulated experimental data and in the scan for parameter reconstruction is the same (R model in the first row and D model in the second). The inner and outer contours are 68 and $99 \%$ confidence levels, respectively. The dot indicates the benchmark value of the parameters, while the encircled cross denotes the position of the best-fit values. The third and fourth rows display the corresponding marginalized pdf. 

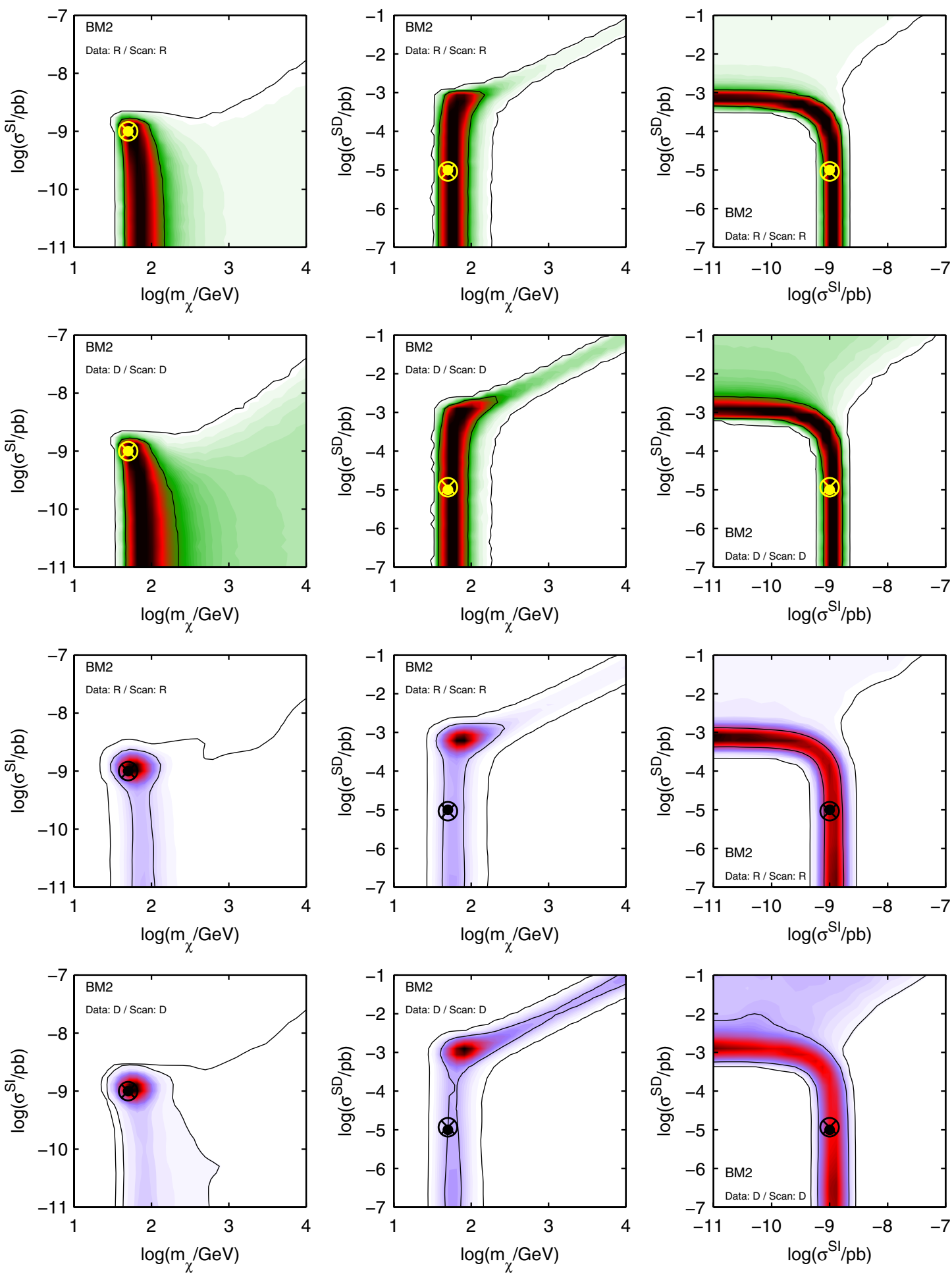

FIG. 5 (color online). The same as in Fig. 4 but for benchmark BM2.

Fig. 2), and we have explicitly checked that it has no influence on the reconstruction of parameters. On the other hand, small differences arise when different SDSF are used in the computation of the likelihood. As already pointed out, the $\mathrm{R}$ and $\mathrm{D}$ models differ in the zero-momentum value, as well as in the slope. Indeed, we find that when the $\mathrm{R}$ model is used in the scan to reconstruct the DM parameters, the resulting $\sigma^{\mathrm{SD}}$ can be smaller than when the D model is used. This happens because the SDSF of the $\mathrm{R}$ model is always larger than in the D model, so the correct number of recoils is reproduced with a slightly smaller $\sigma^{\mathrm{SD}}$. Regarding the reconstructed WIMP mass, 

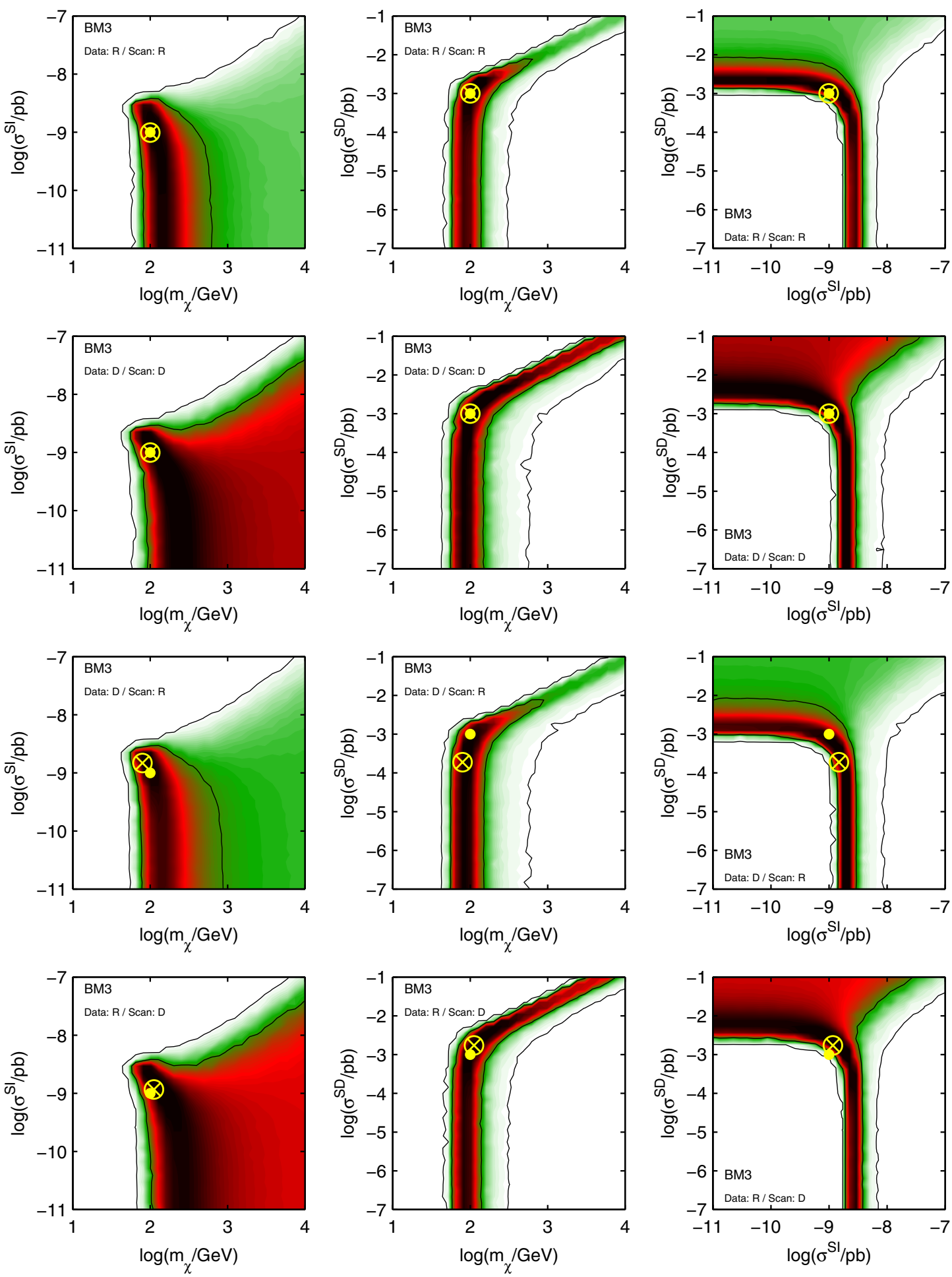

FIG. 6 (color online). The same as in Fig. 4 but for benchmark BM3. Now, in the third row the D model is used for the simulated data and the $\mathrm{R}$ model for the parameter reconstruction; the reverse is done in the fourth row (see the captions of the different panels).

the distribution is similar when either the R or D model is used, although the latter slightly favors heavier WIMPs to compensate for the steeper slope.

Let us now consider the second benchmark, BM2. We proceed as in the previous case and show in Fig. 5 the corresponding reconstruction of the phenomenological parameters in terms of the profile likelihood and pdf. The difference between profile likelihood and pdf (due to the volume effect) is now more striking, especially regarding the SD component and WIMP mass. The regions with a 

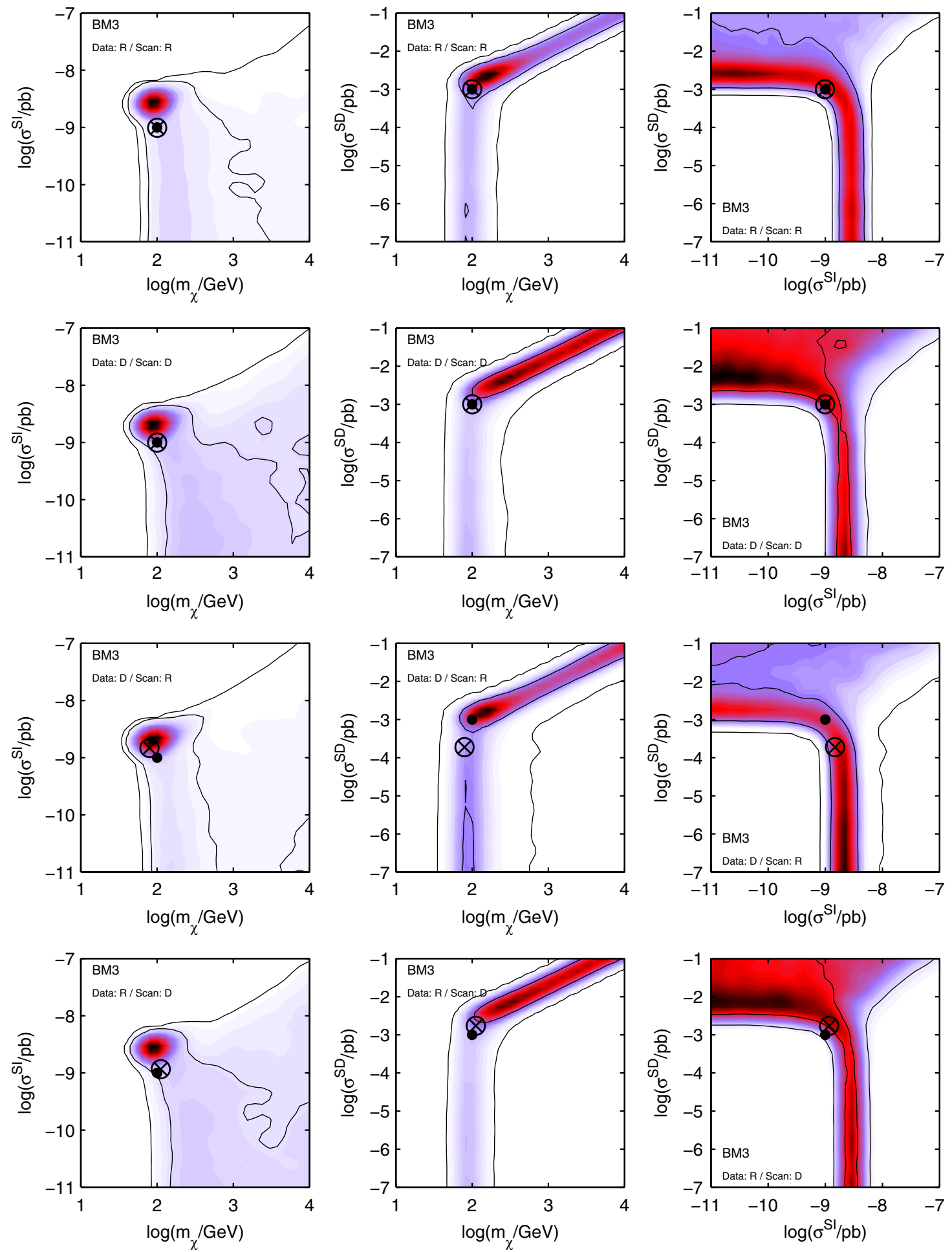

FIG. 7 (color online). The same as in Fig. 7 but for the pdf in benchmark BM3.

best likelihood lie around the correct mass but span many orders of magnitude in $\sigma^{\mathrm{SD}}$. These regions, however, have a small volume and are disfavored when the pdf is plotted. We should emphasize at this point that the information from both sources has a different statistical meaning, and therefore this is not evidence of inconsistency.
As in the previous scenario, the detection rate in this benchmark point is due almost entirely to SI interactions, and there are no differences between the simulated data with either the R or D model for the SDSF (see the middle plot in Fig. 2). However, the number of events is now significantly larger, and this allows a better determination 
of the slope of the recoil spectrum. This has two effects: first, the WIMP mass can be more accurately predicted (points with a heavy WIMP now being more disfavored than in the previous example), and second, it leads to larger differences in the reconstruction of $\sigma^{\mathrm{SD}}$ when different models for the SDSF are used. Notice, for example, how heavy WIMPs are a viable possibility only if the contribution from the SD cross section is sufficiently large (otherwise the shape of the spectrum is not flat enough). In spite of this, the degeneracy between $\sigma^{\mathrm{SI}}$ and $\sigma^{\mathrm{SD}}$ persists. The main difference in the reconstruction using the $\mathrm{R}$ or $\mathrm{D}$ model is again the value of the lowest $\sigma^{\mathrm{SD}}$ compatible with the data (when $\sigma^{\mathrm{SI}}$ is negligible), which is smaller for the $\mathrm{R}$ model. Also, the contours corresponding to the $68 \%$ confidence level extend towards larger WIMP masses in the case of the reconstruction using the D model, in order to compensate for its greater steepness.

Let us finally address the third benchmark point, BM3. Contrary to the previous two cases, the SD cross section is a significant contribution to the total event rate, as we can see in Table I (although it is not entirely dominant). Therefore, we expect that variations in the SDSF play a more important role in the reconstruction of the DM parameters. In fact, small differences arise in the generation of simulated data when a different SDSF is used (see the plot corresponding to BM3 in Fig. 2). For this reason, when displaying the results in Figs. 6 (profile likelihood) and 7 (pdf), we now include two extra rows in which the SDSF model used to simulate data differs from the one used in the scan (see the labels in the plots).

Ignoring the contribution from the SD term is not a good approximation in this scenario. Notice that the reconstruction of $\sigma^{\mathrm{SI}}$ in the limit when $\sigma^{\mathrm{SD}}=0$ is larger (by approximately a factor 2 ) than the nominal value of the BM3 point. This can be observed on the lower parts of the plots in the third column of Fig. 6 (and is consistent with the results of Fig. 3). Moreover, the reconstructed value of $\sigma^{\mathrm{SD}}$ also varies, depending on whether the scan is performed with the R or D model of the SDSF, once more due to the different prediction in the zero-momentum value. As we already noted in the previous benchmark point, an effect in the reconstruction of the WIMP mass can also be observed between these two possibilities. The reconstruction performed with the D model favors heavier masses (in fact, the $68 \%$ confidence level contours of the pdf are open for heavy WIMPs) than those obtained for the R model, since the D model for the SDSF is steeper, and this can be compensated for with a larger value of the WIMP mass, which flattens the spectrum. Notice finally how, although the best-fit value for $\sigma^{\mathrm{SD}}$ is correctly reconstructed when the same SDSF is used for generating and reconstructing the points (first two rows), there is a mismatch when different models are used. For example, if data are generated with the $\mathrm{R}$ model and scanned using the $\mathrm{D}$ model (fourth row), the best-fit value for $\sigma^{\mathrm{SD}}$ is lower than the actual one. Of course, the contrary occurs when data are generated with the $\mathrm{D}$ model and scanned with the $\mathrm{R}$ model (third row).

\section{PARAMETRIZATION OF UNCERTAINTIES IN THE SPIN-DEPENDENT STRUCTURE FUNCTIONS}

In the previous section we have shown that the choice of model for the SDSF has an important effect in the reconstruction of DM parameters. So far, our conclusions are based on the comparison of the results obtained using two different computations for the SDSF of ${ }^{73} \mathrm{Ge}$. In order to consider these effects in a more systematic way, in this section we attempt to include uncertainties in the SDSFs as part of the scan.

To do this, a description of the structure functions has to be found in terms of a relatively small number of parameters. We propose the use of the following family of functions, which reproduces nontrivial features in the shape of SDSFs,

$$
S_{i j}(u)=N\left((1-\beta) e^{-\alpha u}+\beta\right) .
$$

The parameter $N$ acts as an overall normalization that allows us to fit the value at zero momentum, $\beta$ controls the height of a possible tail at large momentum, and $\alpha$ provides the slope of the decreasing part in the lowmomentum regime.

\section{A. Germanium detectors}

In order to account for uncertainties in the SDSFs, we have determined the maximum and minimum values of the three parameters $N, \alpha$, and $\beta$ in Eq. (14) which define an area that contains the calculations of the $\mathrm{R}$ and $\mathrm{D}$ models. The range considered for $S_{11}(q)$ is the following: $N=$ $[0.12,0.21], \beta=[0.020,0.042]$, and $\alpha=[5.0,6.0]$. For illustrative purposes we display in Fig. 1 the area (in blue) spanned by the family of curves that can be obtained by varying the above parameters in the given ranges. As we see, the R and D models correspond approximately to the extremes of the above intervals.

We repeat the scan for each benchmark, extending the parameter space to include $N, \alpha$, and $\beta$. The number of events $\left\{\lambda_{i}\right\}$ of the simulated experimental data is obtained assuming a SDSF with $(N=0.16, \beta=0.031, \alpha=5.5)$ which is located in the center of the above-mentioned ranges. Figure 8 shows the resulting reconstructed contours in the profile likelihood of the DM properties in the three benchmark models. For comparison, we also indicate, by means of blue lines, the contours of the reconstructed DM

\footnotetext{
${ }^{7}$ We have explicitly checked that, although a five-parameter fit is able to better reproduce some features of the SDSF in certain nuclei (e.g., ${ }^{129} \mathrm{Xe}$ and ${ }^{131} \mathrm{Xe}$ ), this has a negligible impact in the reconstruction of DM parameters.
} 

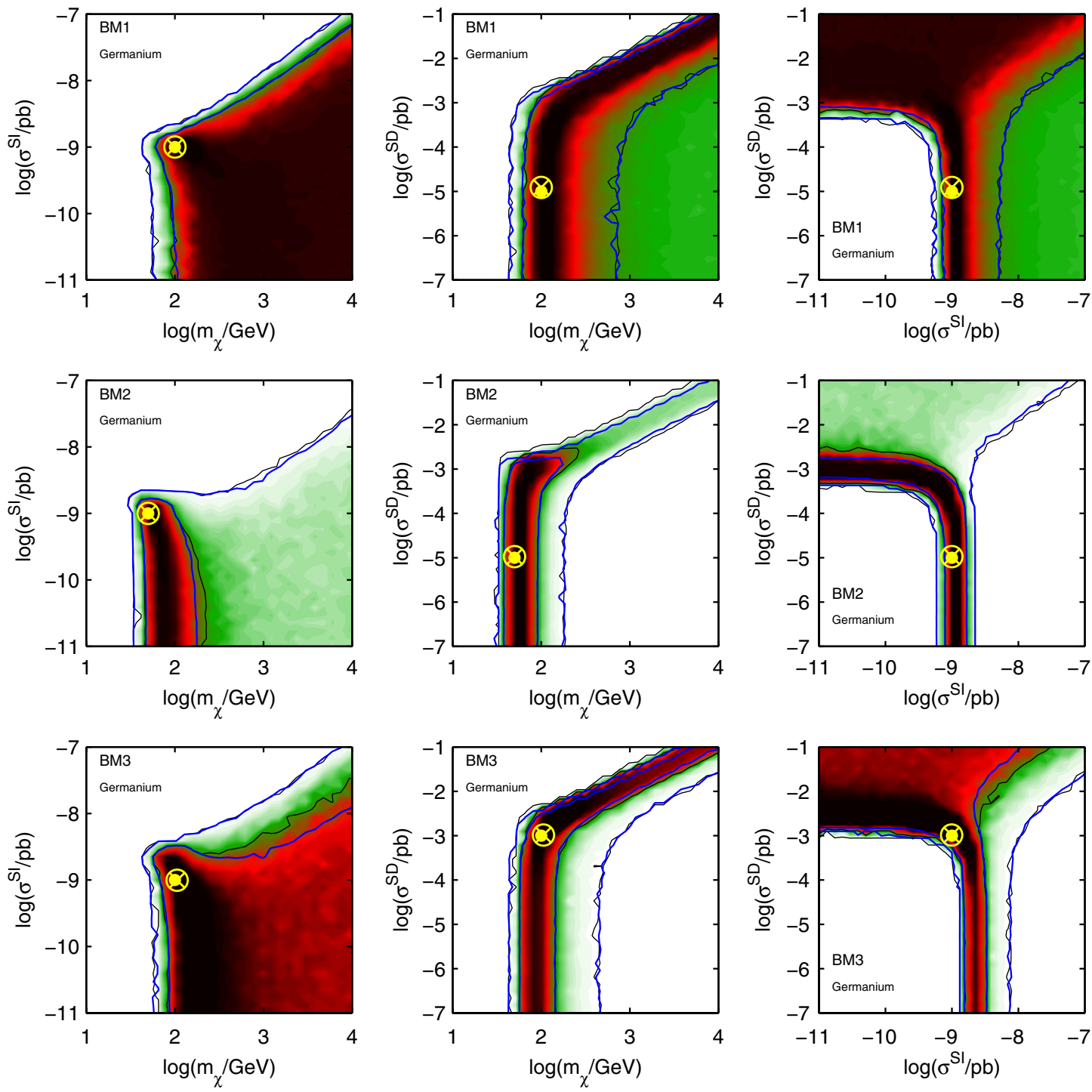

FIG. 8 (color online). Two-dimensional profile likelihood for the reconstructed parameter space $\left(m_{\chi}, \sigma^{\mathrm{SI}}, \sigma^{\mathrm{SD}}\right)$ in benchmark models BM1, BM2, and BM3 (from top to bottom), including nuclear uncertainties in the SDSF through the three-parameter model introduced in Eq. (14). The inner and outer thin black contours are $68 \%$ and $99 \%$ confidence levels, respectively. The solid thick blue line corresponds to the case without uncertainties. The dot indicates the benchmark value of the parameters, while the encircled cross is the position of the best-fit values.

parameters when nuclear uncertainties are not included and where $N, \alpha$, and $\beta$ are fixed to their central values.

We observe that in the case of BM1 the differences with respect to the case with no uncertainties are very small. One can only observe a slight widening in the determination of $\sigma^{\mathrm{SD}}$ when uncertainties in the SDSF are included, but otherwise the reconstructed regions in the parameter space show very little differences. This occurs because in BM1 the DM candidate interacts mainly through SI interactions, and it is thus fairly independent of the details of the SD term. Something similar occurs in the case of BM2, although the widening of the reconstruction of $\sigma^{\mathrm{SD}}$ is more evident now. Also, the $68 \%$ confidence level curves corresponding to the WIMP mass extend to slightly larger values (notice that the logarithmic scale makes this effect more difficult to observe). Finally, it is in benchmark BM3 that the largest effects are found, since the SD contribution is larger. Once more, a widening in the determination of $\sigma^{\mathrm{SD}}$ is observed, which is now more evident in the $68 \%$ confidence level lines. Also, the inclusion of uncertainties in the SDSF enlarges the contours for large WIMP masses.

\section{B. Xenon detectors}

The same procedure can be used for xenon detectors. Natural xenon contains two isotopes, ${ }^{129} \mathrm{Xe}$ (with a $26.4 \%$ isotopic abundance) and ${ }^{131} \mathrm{Xe}(21.29 \%)$, which are 

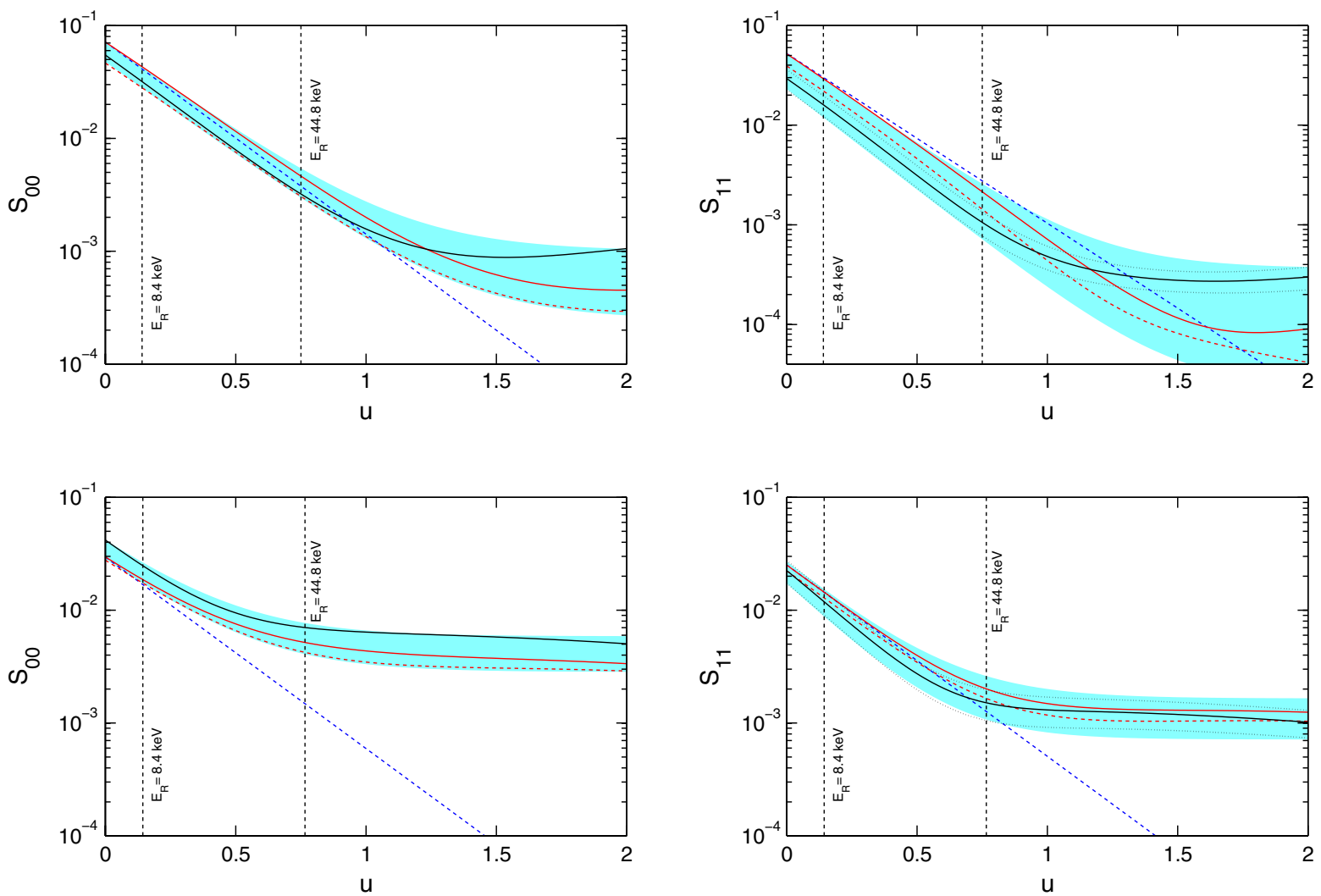

FIG. 9 (color online). The same as in Fig. 1 but for the case of ${ }^{129} \mathrm{Xe}$ (top row) and ${ }^{131} \mathrm{Xe}$ (bottom row). The solid (dashed) gray lines (red) correspond to the ShM calculation using the Bonn A (Nijmegen II) potential [39]. The solid black line corresponds to the determination of Ref. [40], and the dotted black lines are the errors associated with it (the errors for $S_{00}$ are negligible and are not shown). The dotted blue line indicates the Gaussian approximation of Eq. (8). The shaded blue region covers the area spanned by the family of curves in Eq. (14), with the parameters defined in the text. The vertical black dashed lines indicate the WIMP search window used in the analysis.

sensitive to the SD component of the WIMP interaction (in particular, to the SD cross section of the WIMP with neutrons). As in the case of germanium, we consider various parametrizations of the SD form factor for these nuclei from Ref. [39], in which the nuclear shell model was applied to two different potentials describing the nucleonnucleon interaction, the Bonn A [68] and Nijmegen II [69] potentials. We also include a recent result from Ref. [40] in which the so-called gen5082 interaction [70] is used. Then we repeat the analysis of the previous section by modeling the uncertainties in the xenon SDSF by means of the parametrizations in Eq. (14), changing the values of the $(N, \alpha, \beta)$ parameters to define the area that contains the above-mentioned models for the SDSFs. In particular, for the $S_{11}$ component in ${ }^{129} \mathrm{Xe}$ we consider $N=$ $[0.029,0.052], \alpha=[4.2,4.7]$, and $\beta=\left[1.0 \times 10^{-3}, 7 \times\right.$ $\left.10^{-3}\right]$. Similarly, in ${ }^{131} \mathrm{Xe}$ the ranges for $S_{11}$ are $N=[0.017,0.027], \alpha=[4.3,5.0]$, and $\beta=\left[4.2 \times 10^{-2}\right.$, $\left.6.1 \times 10^{-2}\right]$. The various models for the SDSFs are represented in Fig. 9, together with the envelopes for $S_{00}$ and $S_{11}$ in both isotopes. We consider the same exposure as in the previous case $(\epsilon=300 \mathrm{~kg} \mathrm{yr})$, but the energy range of the WIMP detection window is now taken to be $E_{R}=[8.4,44.8] \mathrm{keV}$, mimicking that of the XENON100 experiment.

Uncertainties in the SDSF for xenon have the same qualitative effect as in germanium. Namely, the predictions for the WIMP mass and the SD component of its scattering cross section are affected. The resulting contours for the profile likelihood benchmarks BM1, BM2, and BM3 are displayed in Fig. 10. We can observe that the effect is similar in magnitude to the case of germanium (despite being a heavier nucleus than germanium, the isotopic abundance of the elements sensitive to the SD coupling is larger in xenon). Once more, deviations are larger for BM2 and BM3 than in BM1. Notice that if we had only used the result from Ref. [40] the associated uncertainty in $S_{11}(0)$ would still be sizable (see Fig. 9). In the case of ${ }^{129} \mathrm{Xe}$ it would lead to a slightly reduced range in $N$ (approximately half of what we considered above), but for ${ }^{131} \mathrm{Xe}$ the uncertainty is still so large that it covers all the other models considered and there would be no change in the range in $N$. We would therefore expect only a slightly smaller effect in the parameter reconstruction. 

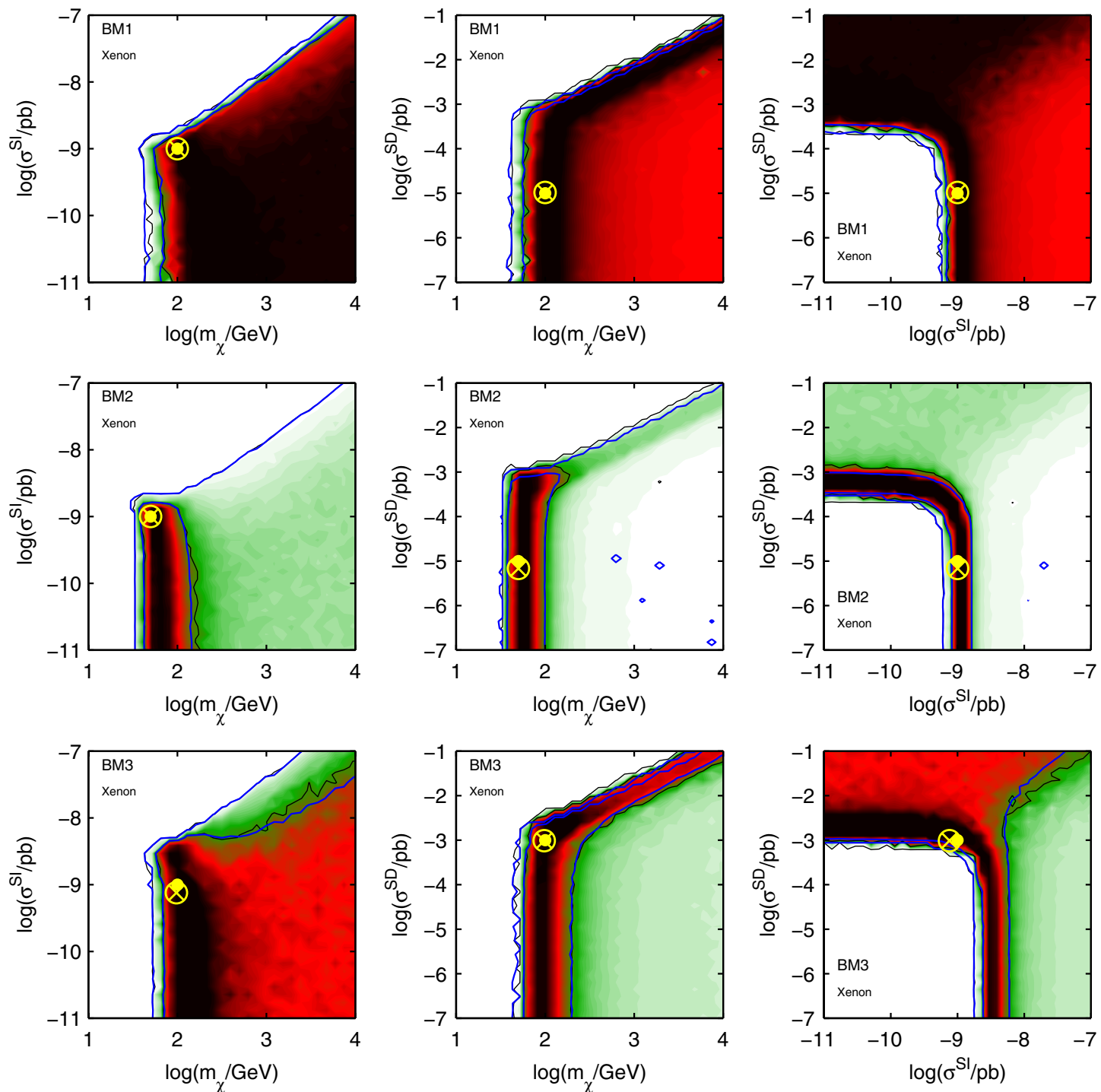

FIG. 10 (color online). The same as in Fig. 8 but for the case of a xenon detector.

This illustrates the importance of a good determination of the nuclear uncertainty associated with the calculation of SDSFs.

The inclusion of uncertainties on SDSF through the parametrization in Eq. (14) is a procedure that can be applied to other nuclei. In the case of germanium and xenon, the existence of different SDSF computations allowed us to define the ranges in which the three parameters of Eq. (14) are varied.

\section{Comparison with astrophysical uncertainties}

To put our results into context, we need to compare the effects of nuclear uncertainties in the SDSF that we just discussed with those originating from astrophysical uncertainties in the parameters of the DM halo. In order to introduce the latter, we have considered a halo model motivated by $\mathrm{N}$-body simulations, which differs from the standard halo model in a high-velocity tail [44,71-73]. The distribution function is taken from Ref. [45], and it is characterized by the presence of an additional parameter $k$ that controls the deviations of $F(v)$ from the standard halo model,

$F(v)=N_{k}^{-1} v^{2}\left[e^{-v^{2} / k v_{0}^{2}}-e^{-v_{\mathrm{esc}}^{2} / k v_{0}^{2}}\right]^{k} \Theta\left(v_{\mathrm{esc}}-v\right)$,

where $N_{k}=v_{0}^{3} e^{-y_{e}^{2}} \int_{0}^{y_{e}} d y y^{2}\left(e^{-\left(y^{2}-y_{e}^{2}\right) / k}-1\right)^{k}$ and $y_{e}=$ $v_{\text {esc }} / v_{0}$. In the limit of vanishing $k$ the standard halo model is recovered. We then consider that the three parameters that define the velocity distribution function vary in the ranges $v_{\text {esc }}[478,610] \mathrm{km} \mathrm{s}^{-1}$, $v_{0}[170,290] \mathrm{km} \mathrm{s}^{-1}$, and $k \in[0.5,3.5]$, and include them in our scan as nuisance parameters. The local DM density is also subject to observational uncertainties. 

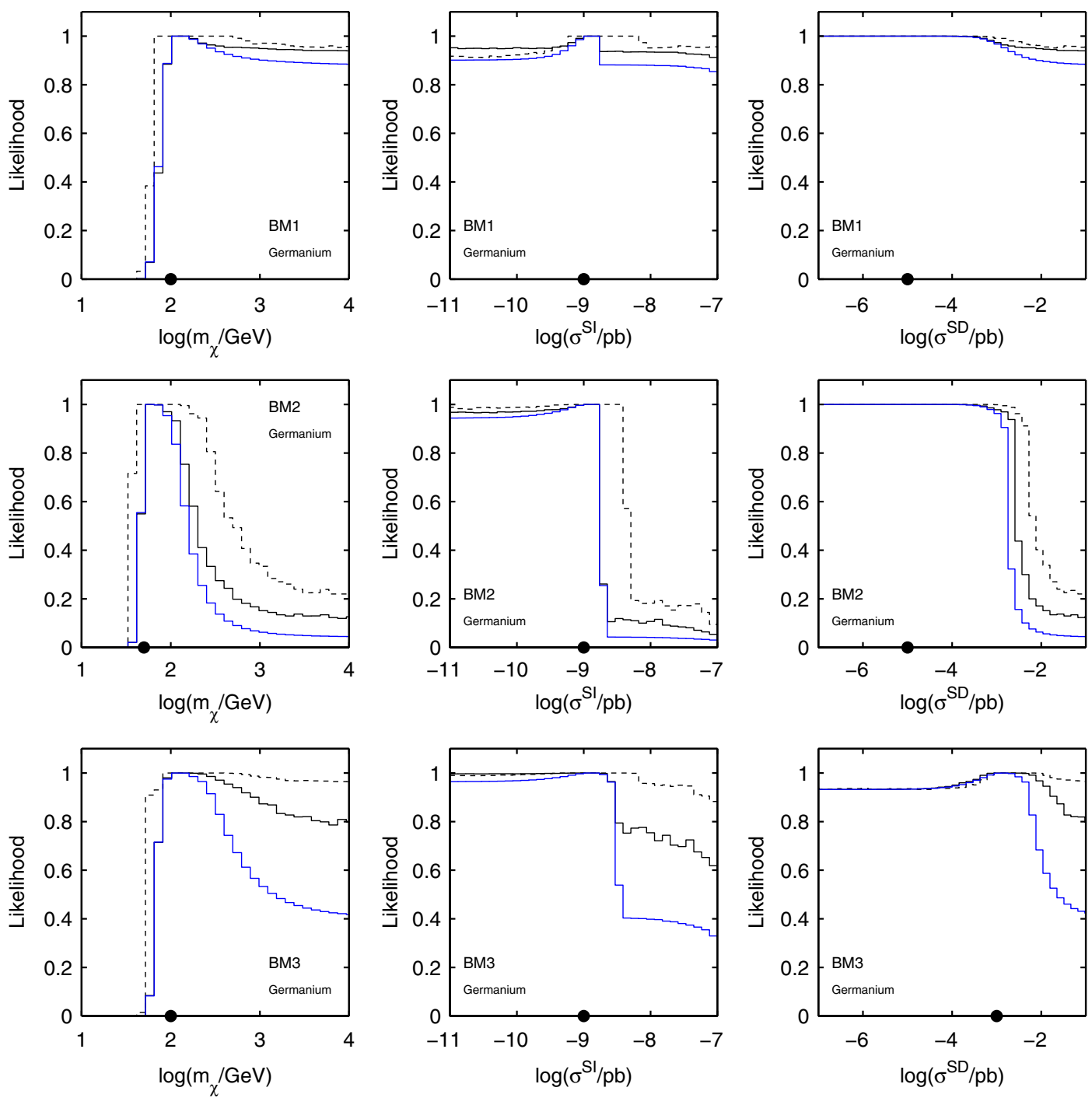

FIG. 11 (color online). One-dimensional profile likelihood for $m_{\chi}, \sigma^{\mathrm{SI}}$, and $\sigma^{\mathrm{SD}}$ in BM1, BM2, and BM3 from top to bottom, respectively, in the case of a germanium detector. From bottom to top, the solid blue line corresponds to the case without uncertainties, the black solid line represents the results when nuclear uncertainties in the SDSF are included, and the dashed black line denotes the case when astrophysical uncertainties are included. The black dot represents the benchmark value of the parameters.

Its value can be estimated from a set of experimental constraints that fix the local gravitational potential of the Milky Way, with typical values ranging from 0.2 to $0.6 \mathrm{GeV} \mathrm{cm}^{-3}[52-55]$.

In Figs. 11 and 12 we represent the one-dimensional profile likelihood for the DM parameters $\left(m_{\chi}, \sigma^{\mathrm{SI}}, \sigma^{\mathrm{SD}}\right)$ for benchmarks BM1, BM2, and BM3 in the cases of germanium and xenon detectors, respectively. We display the reconstruction when no uncertainties are considered (blue line), when only nuclear uncertainties in the SDSF are included (solid black line), and when only astrophysical uncertainties are included (dashed black line). As noted before, the effect of nuclear uncertainties in the SDSF is more evident for BM3 than in BM1 and BM2 in both germanium and xenon, since in the latter the SD component is more important. The prediction for the WIMP mass is extended towards larger masses, and as we see for BM3 the effect cannot generally be neglected. Similarly, the predicted $\sigma^{\mathrm{SD}}$ can vary significantly. In BM3 the reconstruction extends towards larger values (in BM2 and BM1 the effect is smaller). On the other hand, astrophysical uncertainties affect the reconstruction of the three DM parameters, $m_{\chi}, \sigma^{\mathrm{SI}}$, and $\sigma^{\mathrm{SD}}$, and are equally relevant, irrespectively of whether the main contribution comes from the SD or SI component. We can see how nuclear uncertainties generally have a smaller effect than astrophysical ones, but they can be comparable in some benchmark scenarios, especially regarding the mass reconstruction. This is the case, e.g., of BM3 in a xenon detector. 

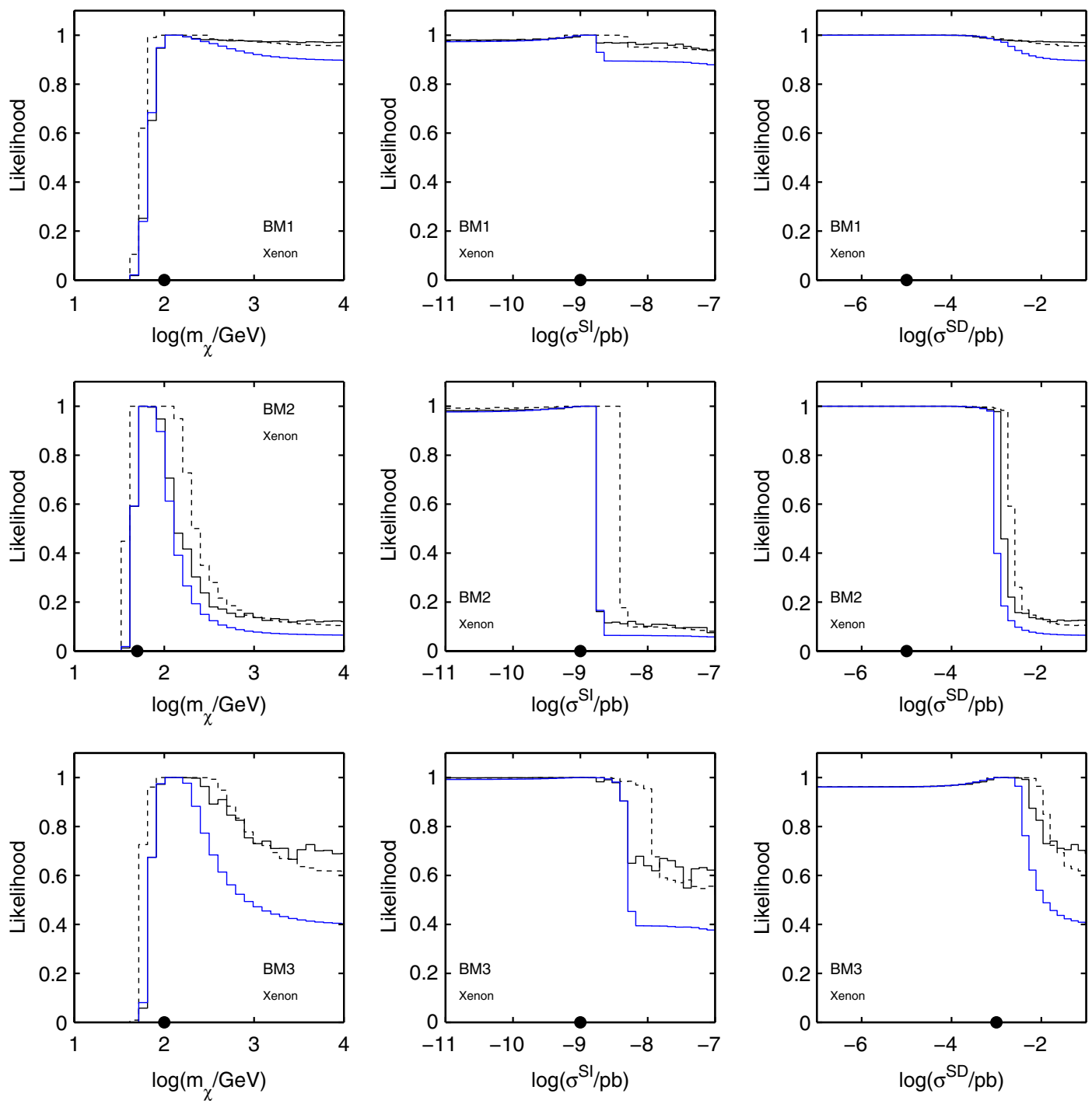

FIG. 12 (color online). The same as in Fig. 11 but for the case of a xenon detector.

\section{CONCLUSIONS}

We have studied the effect that uncertainties in the nuclear spin-dependent structure functions have in the reconstruction of DM properties by means of direct detection experiments.

Assuming a hypothetical future observation of DM in a direct detection experiment, we have systematically investigated how well its phenomenological parameters $\left(m_{\chi}, \sigma^{\mathrm{SI}}, \sigma^{\mathrm{SD}}\right)$ can be determined when uncertainties in the SD form factors of the target nuclei are taken into account. We focused at first on the case of a germanium target and considered two possible models describing the SDSF of its isotope ${ }^{73} \mathrm{Ge}$, sensitive to SD WIMP couplings. Using a Bayesian inference algorithm we determined, for each of these models, the pdf and profile likelihood of the DM parameters in a set of benchmark scenarios. We observed that if a model is chosen to describe the SDSF of a particular nucleus, the reconstruction of the DM properties can strongly depend on the choice made (see, in this sense, the comparison between the predictions using the R or D model in Figs. 6 and 7). In particular, differences in the reconstructed values of the WIMP mass as well as the SD component of the WIMP-nucleon scattering cross section appear. In general, these effects are more important when the SD contribution to the total detection rate is not negligible.

In the second part of the paper we have proposed a description of the SD structure functions in terms of three parameters which fit the zero-momentum value and the slope of the SDSF, and account for the presence of a highmomentum tail. This allows us to include uncertainties in the SDSF in the sampling of the parameter space and treat them in a consistent and systematic way. Using this method we have computed the profile likelihood for the DM parameters for the same three benchmark points as 
before, in the case of a germanium-based and a xenonbased detector.

Finally, we have explicitly compared the effect of nuclear uncertainties in the SDSF with those that are associated with the parameters of the halo of dark matter. We find that uncertainties in the SDSF can even be comparable in magnitude to astrophysical ones when the SD contribution to the total detection rate is sizable.

\section{ACKNOWLEDGMENTS}

We have greatly benefited from discussions with $\mathrm{M}$. Cannoni, P. Gondolo, A. M. Green, B. Kavanagh, and L. Robledo. We are grateful to A. Díaz-Gil for technical support with the computing facilities at the Instituto de Física Teórica and H.D. Kim and the Seoul National
University for allowing us to use their computational facilities in the last stages of the project. D. G.C. is supported by the Ramón y Cajal program of the Spanish MICINN. M. F. is supported by a Leverhulme Trust grant. J.-H.H. is supported by a MultiDark Fellowship. M.P. is supported by a MultiDark Scholarship. This work was supported by the Consolider-Ingenio 2010 Programme under MultiDark Grant No. CSD2009-00064. We also thank the support of the Spanish MICINN under Grants No. FPA2009-08958 and FPA2012-34694, the Spanish MINECO "Centro de excelencia Severo Ochoa Program" under Grant No. SEV-2012-0249, the Community of Madrid under Grant No. HEPHACOS S2009/ESP-1473, and the European Union under the Marie Curie-ITN Program No. PITN-GA-2009-237920.
[1] D. G. Cerdeño and A. M. Green, in Particle Dark Matter, edited by G. Bertone (Cambridge University Press, Cambridge, England, 2010), Chap. 16, pp. 347-369.

[2] R. Bernabei et al., Riv. Nuovo Cimento 26N1, 1 (2003).

[3] R. Bernabei et al. (DAMA Collaboration), Eur. Phys. J. C 56, 333 (2008).

[4] C. E. Aalseth et al. (CoGeNT Collaboration), Phys. Rev. Lett. 106, 131301 (2011).

[5] C. E. Aalseth, P. S. Barbeau, J. Colaresi, J. I. Collar, J. D. Leon, J. E. Fast, N. Fields, and T. W. Hossbach et al., Phys. Rev. Lett. 107, 141301 (2011).

[6] G. Angloher, M. Bauer, I. Bavykina, A. Bento, C. Bucci, C. Ciemniak, G. Deuter, and F. von Feilitzsch et al., Eur. Phys. J. C 72, 1971 (2012).

[7] Z. Ahmed et al. (CDMS-II Collaboration), Science 327, 1619 (2010).

[8] Z. Ahmed et al. (CDMS-II Collaboration), Phys. Rev. Lett. 106, 131302 (2011).

[9] J. Angle et al. (XENON10 Collaboration), Phys. Rev. Lett. 107, 051301 (2011).

[10] E. Aprile et al. (XENON100 Collaboration), Phys. Rev. Lett. 107, 131302 (2011).

[11] http://www.itp.uzh.ch/events/darkattack/talks/Aprile.pdf.

[12] E. Armengaud et al. (EDELWEISS Collaboration), Phys. Lett. B 702, 329 (2011).

[13] M. Felizardo, T. Girard, T. Morlat, A. C. Fernandes, F. Giuliani, A. R. Ramos, J. G. Marques, M. Auguste et al., Phys. Rev. Lett. 108, 201302 (2012).

[14] S. C. Kim, H. Bhang, J. H. Choi, W. G. Kang, B. H. Kim, H. J. Kim, K. W. Kim, and S. K. Kim et al., Phys. Rev. Lett. 108, 181301 (2012).

[15] Z. Ahmed et al. (CDMS and EDELWEISS Collaborations), Phys. Rev. D 84, 011102 (2011).

[16] Z. Ahmed et al. (CDMS Collaboration), arXiv:1203.1309.

[17] J. Angle, E. Aprile, F. Arneodo, L. Baudis, A. Bernstein, A. Bolozdynya, L. C. C. Coelho, and C.E. Dahl et al., Phys. Rev. Lett. 101, 091301 (2008).
[18] E. Behnke, J. Behnke, S. J. Brice, D. Broemmelsiek, J. I. Collar, P. S. Cooper, M. Crisler, and C. E. Dahl et al., Phys. Rev. Lett. 106, 021303 (2011).

[19] S. Archambault et al. (PICASSO Collaboration), Phys. Lett. B 711, 153 (2012).

[20] T. Tanaka et al. (Super-Kamiokande Collaboration), Astrophys. J. 742, 78 (2011).

[21] R. Abbasi et al. (ICECUBE Collaboration), Phys. Rev. Lett. 102, 201302 (2009).

[22] J. Goodman, M. Ibe, A. Rajaraman, W. Shepherd, T. M. P. Tait, and H.-B. Yu, Phys. Lett. B 695, 185 (2011).

[23] J. Goodman, M. Ibe, A. Rajaraman, W. Shepherd, T. M. P. Tait, and H.-B. Yu, Phys. Rev. D 82, 116010 (2010).

[24] T. Aaltonen et al. (CDF Collaboration), Phys. Rev. Lett. 108, 211804 (2012).

[25] A. Rajaraman, W. Shepherd, T.M. P. Tait, and A. M. Wijangco, Phys. Rev. D 84, 095013 (2011).

[26] S. Chatrchyan et al. (CMS Collaboration), J. High Energy Phys. 09 (2012) 094.

[27] P. J. Fox, R. Harnik, J. Kopp, and Y. Tsai, Phys. Rev. D 85, 056011 (2012).

[28] A. M. Green, J. Cosmol. Astropart. Phys. 08 (2007) 022.

[29] A. M. Green, J. Cosmol. Astropart. Phys. 07 (2008) 005.

[30] M. Drees and C.-L. Shan, J. Cosmol. Astropart. Phys.06 (2007) 011.

[31] A. M. Green, J. Cosmol. Astropart. Phys. 10 (2010) 034.

[32] Y. Akrami, C. Savage, P. Scott, J. Conrad, and J. Edsjo, J. Cosmol. Astropart. Phys. 04 (2011) 012.

[33] A. M. Green, Mod. Phys. Lett. A 27, 1230004 (2012).

[34] C. Strege, R. Trotta, G. Bertone, A. H. G. Peter, and P. Scott, Phys. Rev. D 86, 023507 (2012).

[35] Y.-Z. Chen, J.-M. Chen, Y.-A. Luo, H. Shen, and X.-Q. Li, Chinese Phys. C 36, 505 (2012).

[36] V. A. Bednyakov and F. Simkovic, Phys. Part. Nucl. 37, S106 (2006). 
[37] M. T. Ressell, M. B. Aufderheide, S. D. Bloom, K. Griest, G. J. Mathews, and D. A. Resler, Phys. Rev. D 48, 5519 (1993).

[38] V. Dimitrov, J. Engel, and S. Pittel, Phys. Rev. D 51, R291 (1995).

[39] M. T. Ressell and D. J. Dean, Phys. Rev. C 56, 535 (1997).

[40] J. Menéndez, D. Gazit, and A. Schwenk, Phys. Rev. D 86, 103511 (2012).

[41] J. D. Lewin and P. F. Smith, Astropart. Phys. 6, 87 (1996).

[42] M. Pato, L. Baudis, G. Bertone, R. R. de Austri, L.E. Strigari, and R. Trotta, Phys. Rev. D 83, 083505 (2011).

[43] J. D. Vergados, S. H. Hansen, and O. Host, Phys. Rev. D 77, 023509 (2008).

[44] M. Kuhlen, N. Weiner, J. Diemand, P. Madau, B. Moore, D. Potter, J. Stadel, and M. Zemp, J. Cosmol. Astropart. Phys. 02 (2010) 030.

[45] M. Lisanti, L.E. Strigari, J.G. Wacker, and R.H. Wechsler, Phys. Rev. D 83, 023519 (2011).

[46] M. Fairbairn, T. Douce, and J. Swift, arXiv:1206.2693.

[47] F. J. Kerr and D. Lynden-Bell, Mon. Not. R. Astron. Soc. 221, 1023 (1986).

[48] M. J. Reid, K. M. Menten, X. W. Zheng, A. Brunthaler, L. Moscadelli, Y.Xu, B. Zhang, and M. Sato et al., Astrophys. J. 700, 137 (2009).

[49] P. J. McMillan and J. J. Binney, arXiv:0907.4685 [Mon. Not. Roy. Astron. Soc. (to be published)].

[50] J. Bovy, D. W. Hogg and H.-W. Rix, Astrophys. J. 704, 1704 (2009).

[51] C. Savage, K. Freese, P. Gondolo, and D. Spolyar, J. Cosmol. Astropart. Phys. 09 (2009) 036.

[52] R. Catena and P. Ullio, J. Cosmol. Astropart. Phys. 08 (2010) 004.

[53] P. Salucci, F. Nesti, G. Gentile, and C. F. Martins, Astron. Astrophys. 523, A83 (2010).

[54] M. Pato, O. Agertz, G. Bertone, B. Moore, and R. Teyssier, Phys. Rev. D 82, 023531 (2010).

[55] F. Iocco, M. Pato, G. Bertone, and P. Jetzer, J. Cosmol. Astropart. Phys. 11 (2011) 029.
[56] G. Duda, A. Kemper, and P. Gondolo, J. Cosmol. Astropart. Phys. 04 (2007) 012.

[57] D. R. Tovey, R. J. Gaitskell, P. Gondolo, Y. A. Ramachers, and L. Roszkowski, Phys. Lett. B 488, 17 (2000).

[58] M. Cannoni, Phys. Rev. D 84, 095017 (2011).

[59] G. Belanger, F. Boudjema, A. Pukhov, and A. Semenov, Comput. Phys. Commun. 180, 747 (2009).

[60] P. Cushman, Proceedings of the 9th Conference on the Identification of Dark Matter, University of Chicago, Chicago, USA, 2012 (unpublished).

[61] G. Bertone, D. G. Cerdeño, M. Fornasa, R. R. de Austri, C. Strege, and R. Trotta, J. Cosmol. Astropart. Phys. 01 (2012) 015.

[62] F. Feroz, K. Cranmer, M. Hobson, R. R. de Austri, and R. Trotta, J. High Energy Phys. 06 (2011) 042.

[63] R. R. de Austri, R. Trotta, and L. Roszkowski, J. High Energy Phys. 05 (2006) 002.

[64] F. Feroz, M. P. Hobson, and M. Bridges, Mon. Not. R. Astron. Soc. 398, 1601 (2009).

[65] F. Feroz and M. P. Hobson, Mon. Not. R. Astron. Soc. 384, 449 (2008).

[66] G. Belanger, E. Nezri, and A. Pukhov, Phys. Rev. D 79, 015008 (2009).

[67] A. H. G. Peter, Phys. Rev. D 83, 125029 (2011).

[68] M. Hjorth-Jensen, T. T. S. Kuo, and E. Osnes, Phys. Rep. 261, 125 (1995).

[69] V. G. J. Stoks, R. A. M. Klomp, C.P.F. Terheggen, and J. J. de Swart, Phys. Rev. C 49, 2950 (1994).

[70] E. Caurier, J. Menendez, F. Nowacki, and A. Poves, Phys. Rev. Lett. 100, 052503 (2008).

[71] M. Vogelsberger, A. Helmi, V. Springel, S. D. M. White, J. Wang, C.S. Frenk, A. Jenkins, A. Ludlow, and J. F. Navarro, Mon. Not. R. Astron. Soc. 395, 797 (2009).

[72] F. S. Ling, E. Nezri, E. Athanassoula, and R. Teyssier, J. Cosmol. Astropart. Phys. 02 (2010) 012.

[73] M. Fairbairn and T. Schwetz, J. Cosmol. Astropart. Phys. 01 (2009) 037. 